\title{
Design of Six Sigma Supply Chains
}

\author{
D. Garg, Student Member, IEEE, Y. Narahari, Senior Member, IEEE, and N. Viswanadham, Fellow, IEEE
}

\begin{abstract}
Variability reduction and business-process synchronization are acknowledged as keys to achieving sharp and timely deliveries in supply-chain networks. In this paper, we introduce a new notion, which we call six sigma supply chains to describe and quantify supply chains with sharp and timely deliveries, and develop an innovative approach for designing such networks. The approach developed in this paper is founded on an intriguing connection between mechanical design tolerancing and supply-chain lead-time compression. We show that the design of six sigma supply chains can be formulated as a mathematical programming problem, opening up a rich new framework for studying supply-chain design optimization problems. To show the efficacy of the notion and the design methodology, we focus on a design optimization problem, which we call the inventory optimization (IOPT) problem. Given a multistage supply-chain network, the IOPT problem seeks to find optimal allocation of lead time variabilities and inventories to individual stages, so as to achieve required levels of delivery performance in a cost-effective way. We formulate and solve the IOPT problem for a four-stage make-to-order liquid petroleum gas supply chain. The solution of the problem offers rich insights into inventory-service level tradeoffs in supply-chain networks and proves the potential of the new approach presented in this paper.
\end{abstract}

Note to Practitioners - This paper builds a bridge between mechanical design tolerancing and supply-chain management. In particular, the paper explores the use of statistical tolerancing techniques in achieving outstanding delivery performance through variability reduction. Informally, a six sigma supply chain is that which delivers products within a customer specified delivery window, with at most 3.4 missed deliveries per million. The innovations in this paper are the following: 1) to define two performance metrics delivery probability and delivery sharpness to describe the precision and accuracy of deliveries, in terms of process capability indexes $C_{p}, C_{p k}$, and $\left.C_{p m} ; 2\right)$ to formulate the supply-chain design optimization problem using the process capability indices; 3) to suggest an efficient solution procedure for the design optimization problem. The paper presents the case study of a two-echelon distribution network and using the framework developed in the paper shows the role of inventory in controlling lead time variability and achieving six sigma levels of delivery performance.

Index Terms-Cycle time compression, delivery probability (DP), delivery sharpness (DS), Motorola six sigma (MSS) quality, process capability indexes (PCIs), process synchronization, six sigma supply chains, supply-chain lead time, variability reduction.

Manuscript received September 5, 2002; revised June 16, 2003

D. Garg and Y. Narahari are with the Department of Computer Science and Automation, Indian Institute of Science, Bangalore 560 012, India (e-mail: dgarg@csa.iisc.ernet.in; hari@csa.iisc.ernet.in).

N. Viswanadham is with the The Logistics Institute-Asia Pacific (a collaboration between the Georgia Institute of Technology and the National University of Singapore), Singapore 119 260, and also with the Department of Mechanical and Production Engineering, National University of Singapore, Singapore 119260 (e-mail: mpenv@nus.edu.sg).

Digital Object Identifier 10.1109/TASE.2004.829436

\section{INTRODUCTION}

Q UPPLY chains provide the backbone for manufacturing, service, and E-business companies. The supply-chain process is a complex, composite business process comprising a hierarchy of different levels of value-delivering business processes. Achieving superior delivery performance is the primary objective of any industry supply chain. Quick and timely deliveries entail high levels of synchronization among all business processes from sourcing to delivery. This in turn calls for variability reduction all along the supply chain. Variability reduction and business-process synchronization are therefore acknowledged as key to achieving superior levels of delivery performance in supply-chain networks.

\section{A. Motivation}

Lead times of individual business processes and the variabilities in the lead times are key determinants of end-to-end delivery performance in supply-chain networks. When the number of resources, operations, and organizations in a supply-chain increases, variability destroys synchronization among the individual processes, leading to poor delivery performance. On the other hand, by reducing variability all along the supply chain in an intelligent way, proper synchronization can be achieved among the constituent processes. This motivates us to explore variability reduction as a means to achieving outstanding delivery performance. We approach this problem in an innovative way by looking at a striking analogy from mechanical design tolerancing.

Variability reduction is a key idea in the statistical tolerancing approaches that are widely used in mechanical-design tolerancing [1]. A complex supply-chain network is much like a complex electromechanical assembly. Each individual business process in a given supply-chain process is analogous to an individual subassembly. Minimizing defective or out-of-date deliveries in supply chains can therefore be viewed as minimizing tolerancing defects in electromechanical assemblies. This analogy provides the motivation and foundation for this paper.

In statistical-design tolerancing, process capability indexes (PCIs) such as $C_{p}, C_{p k}$, and $C_{p m}$ [2], [3] provide an elegant framework for describing the effects of variability. Best practices such as the Motorola six sigma (MSS) program [4] and Taguchi methods [5] have been extensively used in design tolerancing problem solving. In this paper, we use these popular approaches in a unifying way to address variability reduction, synchronization, and delivery performance improvement in supplychain networks.

\section{B. Contributions}

The contribution of this paper is two fold. First, we recognize the key role of variability reduction and synchronization 
in achieving superior delivery performance in supply-chain networks and explore intriguing connections between statistical-design tolerancing and supply-chain lead time compression. Using this analogy, we introduce the notion of six sigma supply chains. We show that the design of six sigma supply chains can be expressed in a natural way as a mathematical programming problem. This provides an appealing framework for studying a rich variety of design optimization and tactical decision making problems in the supply-chain context.

The second part of the paper proves the potential of the proposed methodology by focusing on a specific design optimization problem which we call the inventory optimization (IOPT) problem. We investigate this problem with the specific objective of tying up design of six sigma supply chains with supply-chain IOPT. Given a multistage supply-chain network, the IOPT problem seeks to find optimal allocation of lead time variabilities and inventories to individual stages, so as to achieve required levels of delivery performance in a cost-effective way. The study uses a representative liquid petroleum gas (LPG) supply-chain network, with four stages: supplier, inbound logistics, manufacturer, and outbound logistics. The results obtained are extremely useful for a supply-chain asset manager to quantitatively assess inventory-service level trade offs. For example, a supply-chain manager for the LPG supply chain will be able to determine the optimal number of LPG trucks to keep at the regional depot (RD) and the optimal way of choosing logistics providers, so as to ensure six sigma delivery of LPG trucks to destinations.

The supply chains for which the methodology discussed in this paper can be categorized as "discrete event dynamical systems." That is, the dynamics of the system is driven by occurrence of discrete events such as arrival of a customer order, arrival of a truck at a logistics hub, dispatch of a truck from a distribution center (DC), etc. All supply chains, regardless of whether they are dealing with discrete parts or continuous processes can be modeled as a discrete event dynamical system. The modeling, analysis, design, and optimization studies depend only on this "discrete event dynamics" of the system. The continuous processes that may constitute individual subsystems are modeled at an aggregate level, by representing their state only at discrete epochs of time and through a stochastic representation of the lead time of the process. What is created here is a "lead time model" which does not need to model the exact dynamics of the underlying production process. We only need to model the starting epoch and completion epoch of the activities.

In our view, the concepts and approach developed in this paper provide a framework in which a rich variety of supplychain design and tactical decision problems can be addressed.

\section{Relevant Work}

The subject matter of this paper falls in the intersection of several topical areas of research. These include: 1) variability reduction and lead time compression techniques for business processes; 2) statistical-design tolerancing, and, in particular, the MSS program; 3) IOPT in supply chains.

Cycle-time compression in business processes using variability reduction is the subject matter of a large number of papers in the last decade. See, for example, the paper by
Narahari et al. [6], where variability reduction is applied to subsystems to achieve lead time reduction of product development projects. Hopp and Spearman, in their book [7], have brought out different ways in which variability reduction can be used in compressing lead times of machining and other business processes. Lead time compression in supply chains (using variability reduction techniques) is the subject of several recent papers, see, for example, Narahari et al. [8].

Statistical-design tolerancing is a mature subject in the design community. The key ideas in statistical-design tolerancing which provide the core inputs to this paper are: 1) theory of PCIs [2], [3], [9]; 2) tolerance analysis and tolerance synthesis techniques [10], [11]; 3) the MSS program [4], [12]; 4) Taguchi methods [5]; 5) design for tolerancing [1], [13].

IOPT in supply chains is the topic of numerous papers in the past decade. Important ones of relevance here are those on multiechelon supply chains [14]-[16]. Variability reduction is a central theme in many of these papers. Recent work by Schwartz and Weng [17] is particularly relevant here. This paper discusses the joint effect of lead-time variability and demand uncertainty, as well as the effect of "fair-shares" allocation, on safety stocks in a four-link just-in-time (JIT) supply chain. Masters [14] develops an optimization model to determine near optimal stock levels for multiechelon distribution inventories. His formulation is similar to what we have discussed as the IOPT model in this paper, although his decision variables are different from those identified here. Ettl et al. [15] develop an inventory-queue model of a multiechelon supply chain with base stock policy followed at each store. Given the bill of materials, the nominal lead times, the demand data, and the cost data, their model generates the base stock level at each store that minimizes the overall inventory capital in the network and guarantees the customer service requirements. in several ways. The volume edited by Tayur et al. [16] also contains several IOPT models in the supply-chain context.

The salient feature of our model which makes it attractive and distinguishes it from all the above discussed models, is the notion of six sigma quality for the end-to-end delivery process. Existing models in the literature consider either the availability of the product to the customer as a criterion for customer service level or probability of delivering the product to the customer within a window as a measure of customer's service level. Away from these classical measurements of customer-service levels in the IOPT problem, we propose a novel approach for customer service level, namely accurate and precise deliveries, which is the primary objective of any modern electronic supply chain.

The paper by Garg et al. [18] contains some of the core ideas of this current paper and can be considered as a preliminary version of the current paper. Another companion paper by the authors [19] looks at a particular design-optimization problem called the variance-pool allocation problem in a detailed way. In contrast to [19], this current paper explores an IOPT problem in multistage supply chains, after proposing a new notion, namely six sigma supply chains.

\section{Outline of the Paper}

The paper is organized in following way. In Section II, we review our work on supply-chain PCIs [18]-[21]. This review 


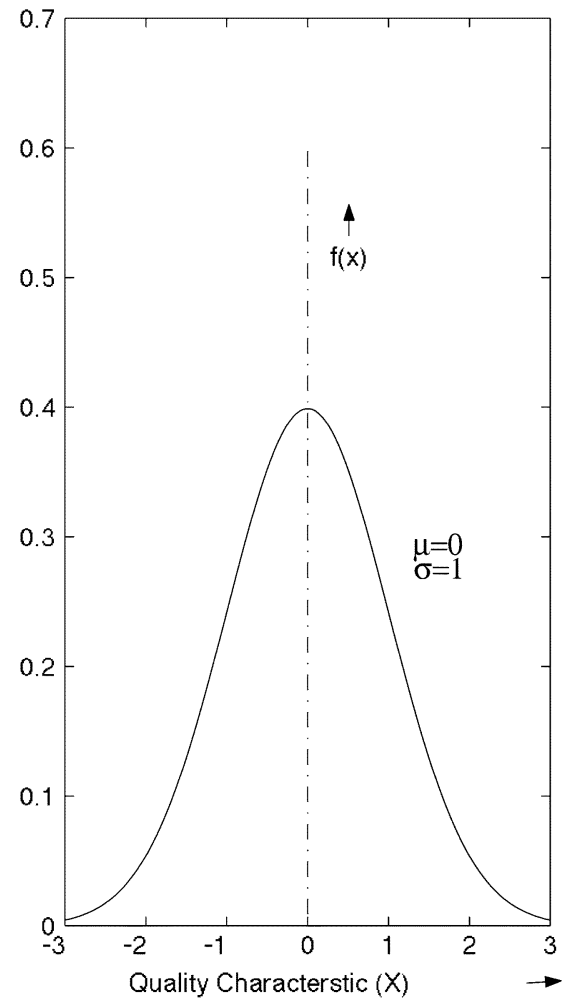

Fig. 1. Process variability and customer delivery window.

is provided for the sake of self-sufficiency of this paper. In this review, we first define PCIs $C_{p}, C_{p k}$, and $C_{p m}$, and show their relevance for modeling the delivery performance of the supplychain process. Next, we present the relationships among these indexes. We define a new performance metric for delivery performance, namely delivery sharpness (DS), to supplement the commonly used metric, delivery probability (DP). Finally, we present an extension of the the MSS quality notion in order to include DS. This prepares the ground for Section III.

In Section III, we first define the notion of six sigma supply chains. Next, we show how the design of a six sigma supply chain can be described in a natural way as a mathematical programming problem. We present several interesting design optimization and tactical decision making studies that can be carried out using this framework.

Section IV is targeted to show the potential of the new approach developed in this paper. We consider a representative four-stage supply-chain network for LPG distribution and formulate a design optimization problem, the IOPT problem, for this network. Given required levels of DP and DS to be achieved, the IOPT problem prescribes the optimal inventory level to be maintained at a designated stage and the optimal way to distribute a pool of variance among lead times of individual stages. Also, we describe a solution procedure for the IOPT problem and present details of the solution approach for a realistic case of the LPG supply chain. Finally, we touch upon numerical results and insights obtained.

Section V provides a summary of contributions of this research and looks at several directions for future work in this important area.

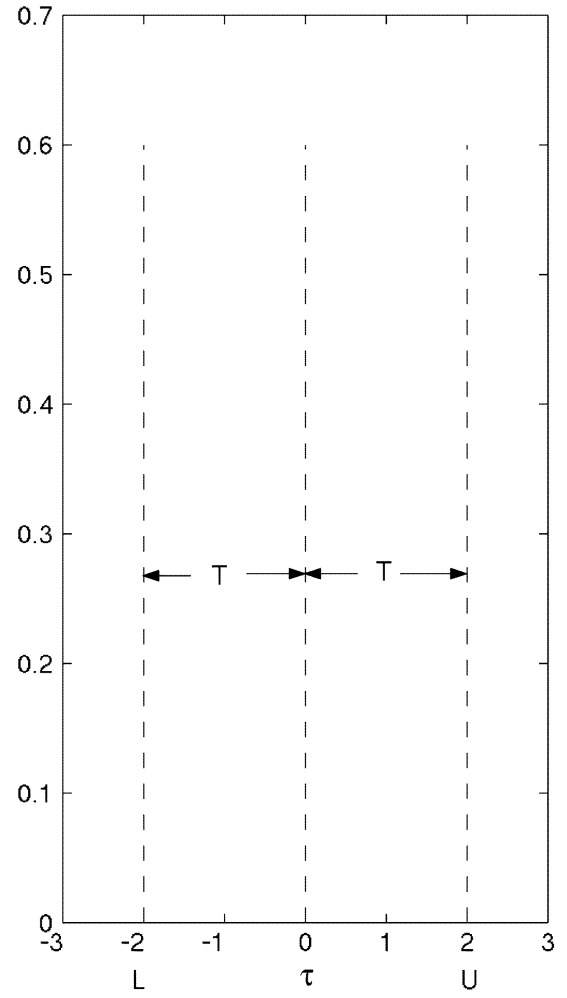

TABLE I

NOTATION USED IN DEFINITIONS OF PCIs

\begin{tabular}{ll}
\hline \hline$X$ & Lead time or any general quality characteristic $X$ \\
$\mu$ & Mean of $X$ \\
$\sigma$ & Standard deviation of $X$ \\
$L$ & Lower specification limit of customer delivery window \\
$U$ & Upper specification limit of customer delivery window \\
$\tau$ & Target value for $X$, specified by customer \\
$\mathrm{T}$ & Tolerance for $X$, specified by customer \\
$b$ & Bias $|\tau-\mu|$ \\
$d$ & $\min (|U-\mu|,|\mu-L|)$ \\
\hline \hline
\end{tabular}

\section{SuPPLY-Chain PCIs}

As already stated, this section is a review of earlier work in [18]-[20].

\section{A. Introduction}

The PCIs $C_{p}, C_{p k}$, and $C_{p m}$ [2] are popular in the areas of design tolerancing and statistical process control. Let us consider the situation depicted by Fig. 1 in order to describe the idea of how capability of a process, where variability is an inherent effect, can be measured. The notation used in Fig. 1 is listed in Table I. In Fig. 1, variability of the process is characterized by the probability density curve of the quality characteristic $X$ produced by the process, and customer specifications are characterized by a delivery window which consists of tolerance $T$ and target value $\tau$. Normal distribution is a popular and common choice for $X$ because of its fundamental role in the theory of PCIs. The target value $\tau$ can be any value between $L$ 

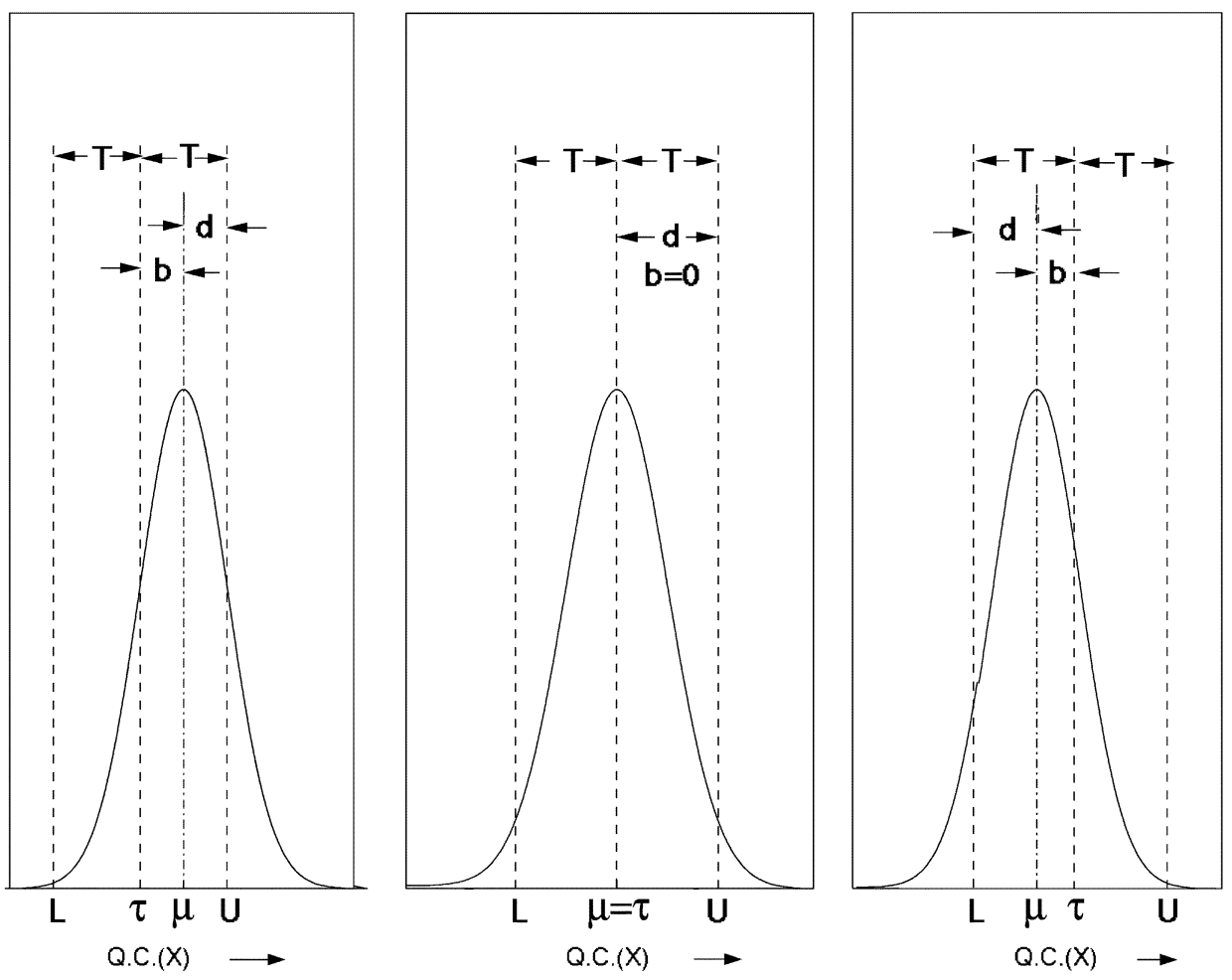

Fig. 2. Process characterization.

and $U$ but we have assumed it as the mid point of two limits for the sake of convenience.

Fig. 2 explains three possible geometries of the probability density curve and customer delivery window when superimposed on each other. This curve is the crux behind the idea of measuring the capability of a process.

\section{B. Indexes $C_{p}, C_{p k}$, and $C_{p m}$}

1) Index $C_{p}$ : The PCI $C_{p}$ is defined as

$$
C_{p}=\frac{U-L}{6 \sigma} \text {. }
$$

As assumed here, the target value $\tau$ is the mid point of $U$, and $L, C_{p}$ can be expressed in the following equivalent form:

$$
C_{p}=\frac{T}{3 \sigma}
$$

where $T=$ tolerance $=(U-L / 2) . C_{p}$ measures only the potential of a process to produce acceptable products. It does not bother about actual yield of the process where potential and actual yield of any process are defined in the following manner.

Actual Yield: The probability of producing a part within specification limits.

Potential: The probability of producing a part within specification limits, if process distribution is centered at the target value i.e., $\mu=\tau$.

It is easy to see [21] that the potential of the process is equal to the area under the probability density curve taken from $X=L$ to $X=U$ when $\mu=\tau$ and it can be expressed by the following relation:

$$
\text { Potential }=2 \Phi\left(3 C_{p}\right)-1
$$

where $\Phi(\cdot)$ is the cumulative distribution function of standard normal distribution.

2) Index $C_{p k}$ : Index $C_{p}$ does not reflect the impact that shifting the process mean or target value has on a process's ability to produce a product within specification [9]. For this reason, the $C_{p k}$ index was developed. $C_{p k}$ is defined as follows:

$$
C_{p k}=\frac{\min (U-\mu, \mu-L)}{3 \sigma}=\left(\frac{d}{3 \sigma}\right) .
$$

Here, $C_{p k}$ alone is not enough to measure actual yield of the process. However, when used with $C_{p}$, it can measure the actual yield of the process. The formula for actual yield can be given as below. A proof for this is provided in [21]

$$
\text { Actual Yield }=\Phi\left(3 C_{p k}\right)+\Phi\left(6 C_{p}-3 C_{p k}\right)-1 .
$$

3) Index $C_{p m}$ : Actual yield of the process is related to the fraction of the total number of units produced by the process which are defective, called as fraction defective. The fraction defective is an indicator of process precision and it does not take into account the accuracy of the process. In order to include the notion of accuracy along with precision, we can use the index $C_{p m}$ [9] as follows:

$$
C_{p m}=\frac{U-L}{6 \sqrt{E(L)}}=\frac{T}{3 \sqrt{\sigma^{2}+b^{2}}} .
$$

The term $E(L)=\sigma^{2}+b^{2}$ is known as the expected Taguchi loss [3].

\section{Relationship and Dependencies Among $C_{p}, C_{p k}$, and $C_{p m}$}

The following relations can be derived among $C_{p}, C_{p k}, C_{p m}$ [21]:

$$
C_{p} \geq C_{p k} \geq 0 \quad C_{p} \geq C_{p m} \geq 0
$$




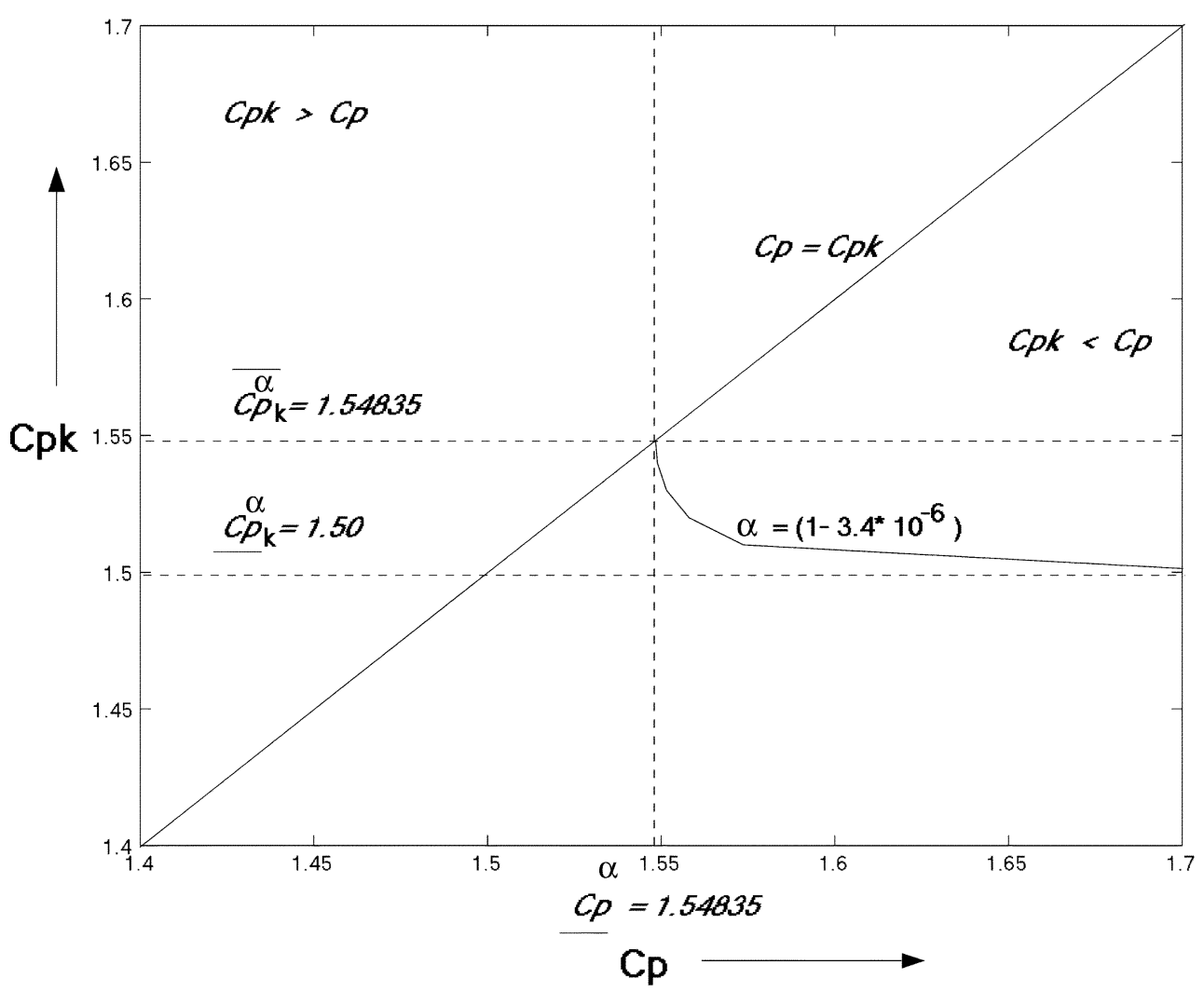

Fig. 3. Typical variation of $C_{p k}$ with respect to $C_{p}$ for constant actual yield.

$$
\begin{aligned}
C_{p k} & =C_{p}(1-k) \text { where } k=\frac{b}{T} \\
\frac{1}{9 C_{p m}^{2}} & =\frac{1}{9 C_{p}^{2}}+\left(1-\frac{C_{p k}}{C_{p}}\right)^{2} .
\end{aligned}
$$

PCIs $C_{p}, C_{p k}$, and $C_{p m}$ have a tight coupling, in addition to the mutual relationship (6), (7), and (8), with process yield. It is easy to show [21] that for a given value of actual yield $\alpha$ (say), one can define lower and upper bounds for the values of both $C_{p}$ and $C_{p k}$. We denote these lower and upper bounds by $C_{p}^{\alpha}, C_{p k}^{\alpha}, \overline{C_{p}^{\alpha}}$, and $\overline{C_{p k}^{\alpha}}$, respectively. A crisp idea behind the intent of these bounds is as follows:

- If the process' $C_{p}\left(C_{p k}\right)$ is less than $C_{p}^{\alpha}\left(C_{p k}^{\alpha}\right)$, then, its actual yield cannot be equal to $\alpha$, no matter how large $C_{p k}\left(C_{p}\right)$ is.

- If the process' $C_{p k}$ is greater than or equal to $\overline{C_{p k}^{\alpha}}$, then, its actual yield cannot be less than $\alpha$, no matter how small $C_{p}$ is.

- The case with $\overline{C_{p}^{\alpha}}$ is a little different. For any value of $C_{p}$ between $\overline{C_{p}^{\alpha}}$ and $C_{p}^{\alpha}$, it is possible to find a corresponding $C_{p k}$ such that the actual yield of the process is $\alpha$.

Table II summarizes such bounds on $C_{p}$ and $C_{p k}$.

Fig. 3 shows a typical variation of $C_{p k}$ with respect to $C_{p}$ when the actual yield $\alpha$ is constant.

Fig. 3 immediately leads to the following observations.

1) For a given pair $\left(C_{p}, C_{p k}\right)$, the value of actual yield is fixed.

2) However, for a given actual yield value, there exist infinite such $\left(C_{p}, C_{p k}\right)$ pairs.
TABLE II

BOUNDS ON PCIs FOR Actual Yield $=\alpha$

\begin{tabular}{ll}
\hline \hline Bound & Formula \\
\hline$\frac{C_{p}^{\alpha}}{3}$ & $\frac{1}{3}\left(\Phi^{-1}\left(\frac{1+\alpha}{2}\right)\right)$ \\
$\frac{C_{p k}^{\alpha}}{\bar{C}}$ & $\frac{1}{3}\left(\Phi^{-1}(\alpha)\right)$ \\
$\overline{C_{p k}^{\alpha}}$ & $\frac{1}{3}\left(\Phi^{-1}\left(\frac{1+\alpha}{2}\right)\right)$ \\
$\overline{C_{p}^{\alpha}}$ & $\infty$ \\
\hline \hline
\end{tabular}

\section{D. $D P$ and $D S$}

For every business process which is a part of a supply-chain process, delivery time of product or service is an important quality characteristic. Variability in lead time is inherent to almost all the business processes, therefore, it will be apt to apply the notion of PCIs to measure the delivery capability or delivery quality of any business process. It is easy to see from the relations presented in the Section II-C, that for a given business process and for a given value of actual yield, there exist infinite pairs $\left(C_{p}, C_{p k}\right)$ such that each one results in the same actual yield. Similarly, by (8), for a given value of $C_{p m}$, there exists an infinite number of feasible $\left(C_{p}, C_{p k}\right)$ pairs. Nevertheless, it can be shown that for a given pair (actual yield $\left.C_{p m}\right)$, there exists a unique feasible pair $\left(C_{p}, C_{p k}\right)$. This suggests that the 3 -tuple $\left(C_{p}, C_{p k}, C_{p m}\right)$ is sufficient to 


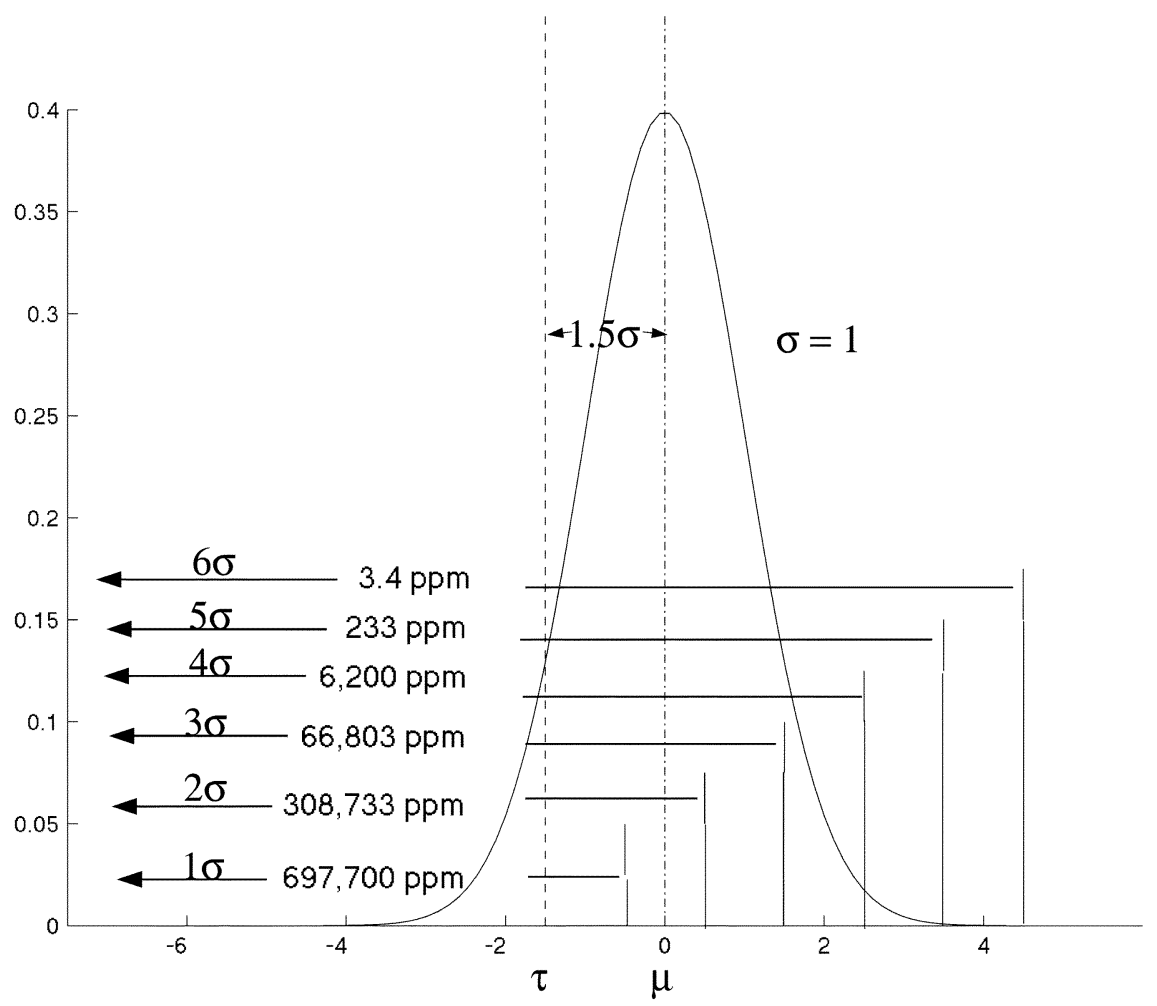

Fig. 4. MSS quality in the presence of shifts and drifts in process mean.

measure the delivery quality of any business process in a given supply chain. This 3-tuple $\left(C_{p}, C_{p k}, C_{p m}\right)$ can be substituted by the pair (actual yield, $C_{p m}$ ) to measure the delivery quality.

In this paper, we use the term $D P$ to refer to the actual yield of a given process. It describes the precision of deliveries. We use the term $D S$ for $C_{p m}$ since it describes the accuracy of deliveries. We use these two indexes to measure the quality of delivery process in a given supply chain. Also, rather than expressing the DP in terms of numerical values, we prefer to express it in terms of $\theta \sigma$ levels where $\theta \in \Re^{+}$. We are motivated by the MSS program [4] in using the idea of $\theta \sigma$ levels. This will be explained in the next section. Each $\theta \sigma$ level corresponds to a particular value of actual yield or equivalently a particular value of number of defects per million opportunities.

\section{E. MSS Quality Program: A Generalized View}

The six sigma concept [4], [12] is a way to measure fractional defectives in a lot. Six sigma quality is the benchmark for excellence of product and process quality. In this concept, a unique $\sigma$ level is attached with each value of number of defects per million opportunities (npmo). The npmo is the probability, expressed on a scale of $10^{-6}$, that a part is produced with quality characteristic $X$ lying outside the specification limits. Here, it is assumed that $X$ is normally distributed and the target value $\tau$ is the midpoint of upper specification limit $(U)$ and lower specification limit $(L)$.

It is not uncommon to the manufacturing processes that $\mu$ begins to drift away from the nominal value of engineering specifications as the machine tool begins to wear and other independent variables such as room temperature, material hardness, etc., come into play. The shifting and drifting of process mean is captured, in the MSS concept, by assuming a one sided mean shift (bias) of $1.5 \sigma$ in the process mean. Also, it is assumed that the variance of the shift in process mean is zero.

The idea behind fixing the value of $\sigma$ levels in this case, is as follows. If $U$ and $L$ coincide with $(\tau+\sigma)$ and $(\tau-\sigma)$ respectively, which are different from $(\mu+\sigma)$ and $(\mu-\sigma)$ (see Fig. 4), then, the corresponding upper bound on yield will be assigned $1 \sigma$ level. Similar is the case with $2 \sigma, 3 \sigma$, and others. Note that the MSS program uses upper bound on yield (not actual yield) in order to assign $\sigma$ levels. However, we follow a slightly different approach. We say DP of the process is $6 \sigma$ iff actual yield of the process is $\left(1-3.4 \times 10^{-6}\right)$ whereas according to the MSS concept, six sigma quality is achieved when the the upper bound of yield is $\left(1-3.4 \times 10^{-6}\right)$.

As shown before, for a given $\left(C_{p}, C_{p k}\right)$ pair, the value of actual yield is fixed. But for a given actual yield value, there exist infinite such $\left(C_{p}, C_{p k}\right)$ pairs. Hence, DP can be completely determined by knowing $C_{p}$ and $C_{p k}$. However, there are numerous (in fact, infinitely many) ways in which we can choose the pair $\left(C_{p}, C_{p k}\right)$ to achieve a given value of DP. This leads to a generalized view of six sigma quality. MSS is a special case of this in which bias is fixed i.e., $1.5 \sigma$. In order to elaborate this idea let us start with the equation

$$
\text { Actual Yield }=\Phi\left(3 C_{p k}\right)+\Phi\left(6 C_{p}-3 C_{p k}\right)-1 .
$$

If we fix the value of the actual yield as $\alpha$ in the above equation, there will be two independent variables $C_{p}, C_{p k}$, and the solution set will be unbounded. However, we have earlier shown that for a given actual yield $\alpha, C_{p}$, and $C_{p k}$ are bounded within certain range. Therefore, the solution is bounded by $\underline{C_{p k}^{\alpha}} \leq C_{p k} \leq$ $\overline{C_{p k}^{\alpha}} ; C_{p}^{\alpha} \leq C_{p} \leq \infty$. If we substitute $\alpha=\left(\overline{1-3.4} \times 10^{-6}\right)$ 


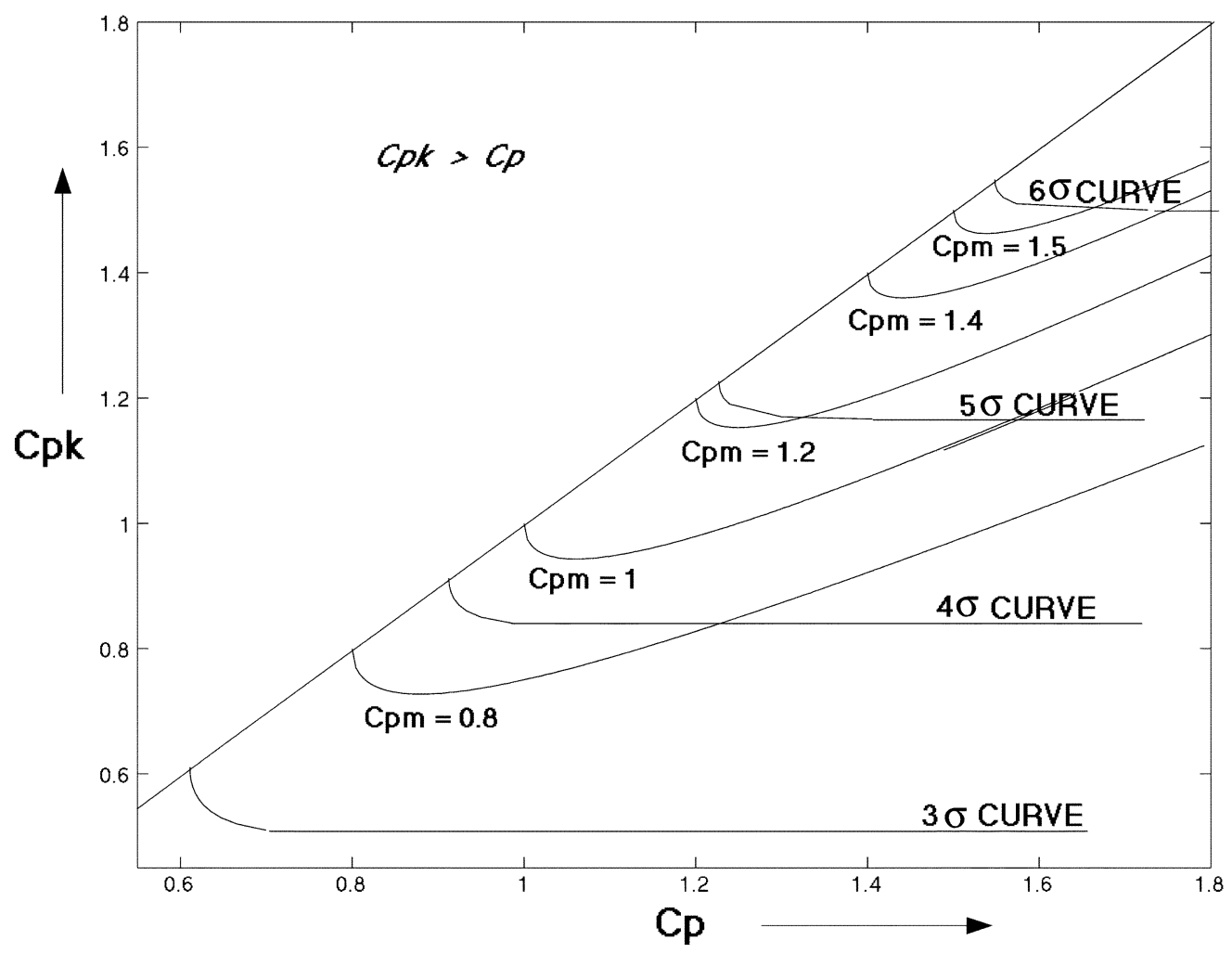

Fig. 5. The $\theta \sigma$ curves and $C_{p m}$ curves on $C_{p k}-C_{p}$ plane.

and plot a graph, then, all the points lying on the curve will give $\left(C_{p}, C_{p k}\right)$ pairs that result in $6 \sigma$ quality level. This equation can be generalized for any $\theta \sigma$ level by expressing $\alpha$ in terms of $\theta$. It is easy to see from Fig. 4 that the upper bound in the MSS program for $\theta \sigma$ level is $\Phi(\theta-1.5)$. Equating this to the actual yield of the process we get the following equation for $\theta \sigma$ quality curve on the $C_{p}-C_{p k}$ plane, we get

$$
\Phi(\theta-1.5)=\Phi\left(3 C_{p k}\right)+\Phi\left(6 C_{p}-3 C_{p k}\right)-1 .
$$

Some of these curves are plotted in Fig. 5. We can proceed one step further by looking at the connection between DP and DS in the light of our generalized notion of six sigma quality. For this, we consider the plots of $\sigma$ quality levels on $C_{p}-C_{p k}$ plane and then see how $C_{p m}$ varies on the same plot. To see this, we use the identity relation (8) among $C_{p}, C_{p k}$, and $C_{p m}$ and plot this relation for a constant value of $C_{p m}$ (say $C_{p m}^{*}$ ). The plot comes out to be a section of a hyperbola. From a process design point of view, it can be said that for a desired level of DS (i.e., $C_{p m}$ ) and DP (i.e., $C_{p}, C_{p k}$ ), this curve provides a set of 3-tuples $\left(C_{p}, C_{p k}, C_{p m}\right)$ which all satisfy these two requirements. The designer has to decide which one of the triples to choose depending upon the requirements. Fig. 5 shows some $C_{p m}$ curves on the $C_{p}-C_{p k}$ plane.

\section{Six Sigma Supply Chains}

\section{A. Notion of Six Sigma Supply Chains}

Motivated by the discussion in the previous section, we seek to define the notion of six sigma supply chains, to describe a supply chain with superior delivery performance. We define a six sigma supply chain as a network of supply-chain elements
TABLE III

Sample Values of 3 -Tuples $\left(C_{p}, C_{p k}, C_{p m}\right)$ Which ACHIEVE Six Sigma Delivery PERFormance

\begin{tabular}{ccc}
\hline \hline$C_{p}$ & $C_{p k}$ & $C_{p m}$ \\
\hline 1.548350 & 1.548350 & 1.548350 \\
1.548900 & 1.540000 & 1.548348 \\
1.551535 & 1.530000 & 1.548307 \\
1.557998 & 1.520000 & 1.547972 \\
1.573665 & 1.510000 & 1.545724 \\
1.721814 & 1.500010 & 1.433445 \\
1.726667 & 1.5000000001 & 1.427826 \\
\hline \hline
\end{tabular}

which, given the customer specified window and the target delivery date, results in defective deliveries (i.e., DP) not more than $3.4 \mathrm{ppm}$. All triples $\left(C_{p}, C_{p k}, C_{p m}\right)$ that guarantee an actual yield of at least $3.4 \mathrm{ppm}$ (or DP $=6 \sigma$ ) would correspond to a six sigma supply chain.

Table III provides sample values of PCIs that achieve six sigma delivery performance. It is important to note that in order to achieve DP $=6 \sigma$, the DS needs to assume appropriately high value. In a given setting, however, there may be a need for extremely sharp deliveries (highly accurate deliveries) implying that the $C_{p m}$ index is required to be very high. This can be specified as an additional requirement of the designer.

\section{B. Design of Six Sigma Supply Chains}

We can say that the design objective in supply-chain networks is to deliver finished products to the customers within a time as close to the target delivery date as possible, with as few defective deliveries as possible at the minimum cost. To give an idea 
of how the design problem of a complex supply-chain network can be formulated, let us consider a supply chain with $n$ business processes such that each of them contributes to the order-to-delivery cycle of customer desired products. Let $X_{i}$ be the cycle time of process $i$. It is realistic to assume that each $X_{i}$ is a continuous random variable with mean $\mu_{i}$ and standard deviation $\sigma_{i}$. The order-to-delivery time $Y$ can then be considered as a deterministic function of $X_{i}$ 's

$$
Y=f\left(X_{1}, \ldots, X_{n}\right) .
$$

If we assume that the cost of delivering the products depends only on the first two moments of these random variables, the total cost of the process can be described as

$$
Z=g\left(\mu_{1}, \sigma_{1}, \ldots, \mu_{n}, \sigma_{n}\right)
$$

where $g$ is some deterministic function.

The customer specifies a lower specification limit $L$, an upper specification limit $U$, and a target value $\tau$ for this order-to-delivery lead time. With respect to this customer specification, we are required to choose the parameters of $X_{1}, \ldots X_{n}$ so as to minimize the total cost involved in reaching the products to the customers, achieving a six sigma level of delivery performance.

Thus, the design problem can be stated as the following mathematical programming problem:

$$
\begin{aligned}
& \text { Minimize } Z=g\left(\mu_{1}, \sigma_{1}, \ldots, \mu_{n}, \sigma_{n}\right) \\
& \text { subject to } \\
& \text { DS for order-to-delivery time } \geq C_{p m}^{*} \\
& \text { DP for order-to-delivery time } \geq 6 \sigma \\
& \mu_{i}>0 \quad \forall i \\
& \sigma_{i}>0 \quad \forall i
\end{aligned}
$$

where $C_{p m}^{*}$ is a required lower bound on DS. The objective function $Z$ of this formulation captures the total cost involved in taking the product to the customer, going through the individual business processes. We have assumed that this cost is determined by the first two moments of lead times of the individual business processes. One can define $Z$ in a more general way if necessary. The decision variables in this formulation are means and/or standard deviations of individual processes. The constraints of this formulation guarantee a minimum level of DS $\left(C_{p m}^{*}\right.$ is the minimum level of DS required) and at least a six sigma level of DP.

While solving the design problem, an important step is to express the constraints in terms of the decision variables. This will be elaborated upon in the next section.

\section{Representative Design Problems}

Depending on the nature of the objective function and decision variables chosen, the six sigma supply-chain design problem assumes interesting forms. We consider some problems below under two categories: 1) generic design problems; 2) concrete design problems.

\section{1) Generic Design Problems:}

- optimal allocation of process means;

- optimal allocation of process variances;

- optimal allocation of customer windows.

\section{2) Concrete Design Problems:}

- due date setting;

- choice of customers;

- inventory allocation;

- capacity planning;

- vendor selection;

- choice of logistics modes, logistics providers;

- choice of manufacturing control policies.

These problems can arise at any level of the hierarchical design. Thus, in order to develop a complete suite for designing a complex supply-chain network for six sigma delivery performance through the hierarchical design scheme, we need to address all such subproblems beforehand. In the Section IV, we consider one such subproblem, optimal allocation of inventory in a multistage six sigma supply chain, and develop a methodology for this problem.

\section{INVENTORY OPTIMIZATION IN A Multistage Supply Chain}

In this section, we describe a representative supply-chain example for LPG, with four stages: supplier (refinery), inbound logistics, manufacturer [regional depot (RG) for LPG], and outbound logistics [17]. We formulate the six sigma design problem, based on the concepts developed in earlier sections, for this supply chain. Then we show how one can allocate variabilities to lead times of individual stages so as to achieve six sigma delivery performance. We also show how to compute the optimal inventory to be maintained at the RD to support six sigma delivery performance.

\section{A. Four-Stage Supply-Chain Model With Demand and Lead Time Uncertainty}

1) Model Description: Consider $N$ geographically dispersed DCs supplying retailer demand for some product as shown in Fig. 6 . The product belongs to a category which does not make it profitable for the DC to maintain any inventory. An immediate example is a distributor who supplies trucks laden with bottled LPG cylinders (call these as LPG trucks or finished product now onward) to retail outlets and industrial customers. In a situation like this, as soon as a demand for an LPG truck arrives at any DC, the DC immediately places an order for one unit of product (in this case, an LPG truck) to a major RD. The $\mathrm{RD}$ maintains an inventory of LPG trucks and after receiving the order, if on-hand inventory of LPG trucks is positive, then, an LPG truck is sent to the DC via outbound logistics. On the other hand, if the on-hand inventory is zero, the order gets backordered at the RD. At the RD, the processing involves unloading the LPG from LPG tankers into LPG reservoirs, filling the LPG into cylinders, bottling the cylinders and finally loading the cylinders onto trucks.

The inventory at RD is replenished as follows. The RD starts with on-hand inventory $R$ and every time an order is received, it places an order to the supplier for one LPG tanker (called the semifinished product now onward) which is sufficient to produce one LPG truck. In this case, the supplier corresponds to a refinery which will produce LPG tankers. In the literature such a replenishment model is known as the $\langle Q, R\rangle$ model [22] with 


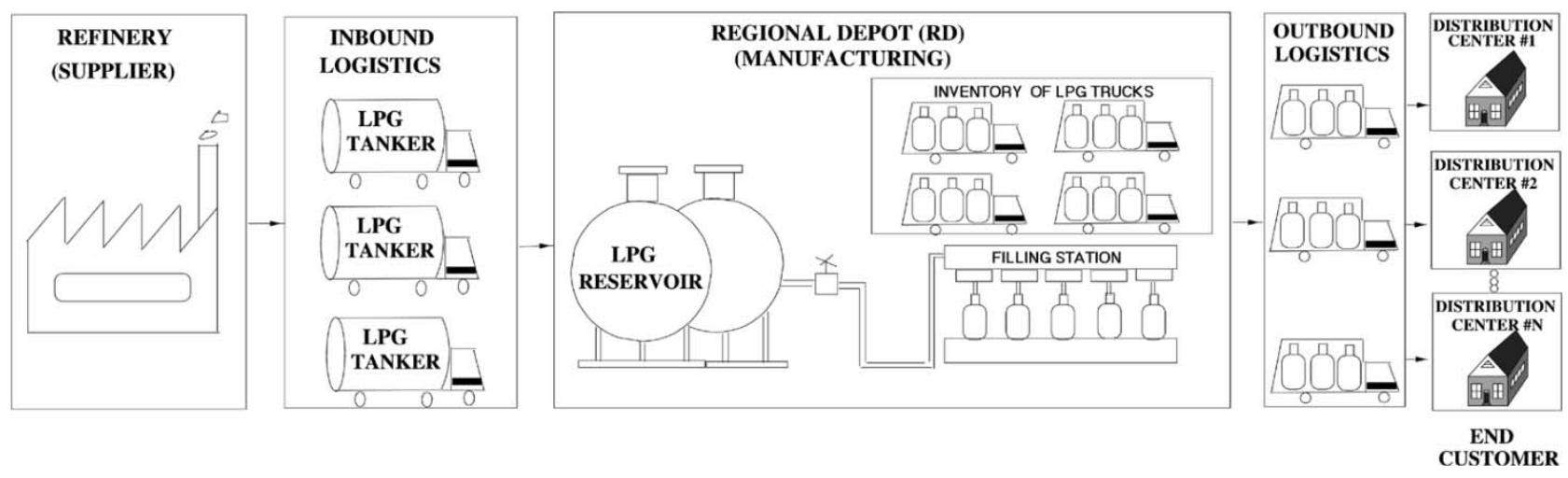

Fig. 6. Four-link linear supply-chain model.

$Q=1$. In such a model, the inventory position (on-hand plus on-order minus backorders) is always constant and is equal to $R$.

It is assumed that raw material (crude oil or naphtha) required for producing an LPG tanker is always available with the refinery, but the refinery needs to do some processing of this raw material to transform it to LPG and load it onto a tanker. Therefore, as soon as the refinery receives an order from the RD, it starts processing the raw material and sends an LPG tanker via inbound logistics to the RD.

The LPG supply-chain network is a typical example of a multiechelon supply-chain network. The four stages could be considered generically as procurement, inbound logistics, manufacturing, and outbound logistics, as described below (descriptions in parentheses corresponds to the LPG example).

1) procurement or supplier (refinery);

2) inbound logistics (transportation of LPG tankers from refinery to RD);

3) manufacturing (RD);

4) outbound logistics (customer order processing and transportation of LPG trucks from RD to a DC).

The DCs in the LPG example correspond to the end user in the general setting of a four-stage supply-chain network. This example emphasizes the distribution aspect of the supply chain.

In the next section, we articulate all the assumptions we have made regarding behavioral and operational characteristics of this model. We believe that these assumptions are reasonable enough to make the model realistic and yet tractable, preserving all important insights of the problem.

2) Assumptions:

1) A customer places order for only one unit of finished product at a time to the manufacturer.

2) The orders arrive at the manufacturer in Poisson fashion from each customer. The Poisson arrival streams of orders are independent across the customers.

3) Each customer specifies a delivery window while placing an order. This window is assumed to be the same for all the customers. Also, in this window the date which customer targets for delivery of the item has equal offset from the upper specification limit and lower specification limit.

4) If the item is not on-hand with the manufacturer, then, the customer's order gets backordered there. All such backorders are fulfilled in a first in first out (FIFO) manner by the manufacturer.
5) Lead time for an item at each stage of the supply chain is a normal random variable. Lead times of the four stages are mutually independent. As soon as a supplier receives an order from the manufacturer, processing commences on the corresponding raw material.

6) Inbound and outbound logistics facilities are always available. Therefore, as soon as an item finishes its processing at the supplier, its shipment starts via the inbound logistics. Similarly, as soon as an order of a customer is received by the manufacturer, the shipment of an item, if available, commences using the outbound logistics. Otherwise the shipment commences as soon as it becomes available at the manufacturing node (following an FIFO policy). Inbound logistics lead times are independ identically distributed (i.i.d.) random variables and outbound logistics lead times are also i.i.d. random variables.

7) An item that arrives from the supplier does not wait in queue at the manufacturer for getting processed (there is no queuing before the manufacturing stage). Processing times for items at the manufacturing node are i.i.d. random variables.

8) The processing cost per item at each stage depends only on the mean and variance of lead time of the stage.

9) Costs related to maintenance of inventory at the manufacturing node are fixed. Such costs include order placing cost, inventory carrying cost, cost of raw material of an item, and fixed cost against backorder of an item. However, the variable cost of the backorder is a function of time for which the order is backordered.

Note that in this example, the following hold.

- We have modeled the dynamics of each of the four individual stages by a corresponding normally distributed lead time random variable.

- We have assumed only one stock, at the RD. We could have assumed inventories at the other three stages, but that would not add much to the insights sought to be illustrated here.

- We have modeled the demand process.

- We have modeled stockouts, backordering, and inventory replenishments corresponding to the inventory at the RD.

3) System Parameters: This section presents the notation used for various system parameters. 
Lead Time Parameters:

$X_{1} \sim N\left(\mu_{1}, \sigma_{1}^{2}\right)$ Procurement lead time.

$X_{2} \sim N\left(\mu_{2}, \sigma_{2}^{2}\right)$ Inbound logistics lead time.

$X_{3} \sim N\left(\mu_{3}, \sigma_{3}^{2}\right)$ Manufacturing lead time.

$X_{4} \sim N\left(\mu_{4}, \sigma_{4}^{2}\right)$ Outbound logistics lead time.

$L_{m}$

$L_{f}$

$\frac{L_{c}}{L_{c}}$

$\overline{L_{c}}$

$\left(\mu_{m}, \sigma_{m}^{2}\right)$.

$\left(\mu_{f}, \sigma_{f}^{2}\right)$

$\left(\mu_{c}, \sigma_{c}^{2}\right)$

$\left(\bar{\mu}_{c},{\overline{\sigma_{c}}}^{2}\right)$

Time elapsed between placement of an order by manufacturer and receipt of the order from supplier.

Demand Process Parameters:

$\lambda_{i} \quad$ Order arrival rate from $i$ th customer (item/year).

$\lambda=\sum_{i}^{N} \lambda_{i}$, Poisson arrival rate of orders at the manufacturer.

$R \quad$ Inventory level at the manufacturing node.

$Q=1$ Reorder quantity of the manufacturer.

$M_{O} \quad$ Stockout probability at the manufacturing node.

$E \quad$ Average number of backorders per unit time at the manufacturing node (item/time).

$B \quad$ Expected number of backorders with the manufacturer at arbitrary time $t$ (item).

$D \quad$ Expected number of on-hand inventory with the manufacturer at arbitrary time $t$ (item).

$\psi_{m}(x)$ Steady-state probability that the manufacturer has a net inventory equal to $x$.

$p(x ; \lambda t)\left(\exp (-\lambda t)(\lambda t)^{x}\right) /(x !)$.

$P\left(r ; \lambda t \sum_{x=r}^{\infty} p(x ; \lambda t)\right.$.

Cost Parameters:

$\mathcal{K}_{1}=f_{1}\left(\mu_{1}, \sigma_{1}\right)$, Procurement cost (\$/item).

$\mathcal{K}_{2} \quad=f_{2}\left(\mu_{2}, \sigma_{2}\right)$, Inbound logistics cost (\$/item).

$\mathcal{K}_{3} \quad=f_{3}\left(\mu_{3}, \sigma_{3}\right)$, Manufacturing cost $(\$ /$ item $)$.

$\mathcal{K}_{4} \quad=f_{4}\left(\mu_{4}, \sigma_{4}\right)$, Outbound logistics cost (\$/item).

A Order placing cost for manufacturer (\$/order).

$\Pi \quad$ Fixed part of backorder cost (\$/item).

$\hat{\Pi} \quad$ Variable part of backorder cost (\$/item-time).

$I \quad$ Inventory carrying cost (\$/time-invested).

$C \quad$ Cost of raw material(\$/item).

$C_{m} \quad$ Capital tied up with each item ready to be

shipped via outbound logistics (\$/item).

Delivery Quality Parameters:

$C_{p}, C_{p k}, C_{p m}$ Supply-chain PCIs for end-to-end lead time of customer order.

$(\tau, T) \quad$ Delivery window specified by customer.

$U=\tau+T \quad$ Upper limit of delivery window.

$L=\tau-T \quad$ Lower limit of delivery window.

$b=\left|\tau-\mu_{c}\right| \quad$ Bias for $L_{c}$.

$\bar{b}=\left|\tau-\overline{\mu_{c}}\right|$. Bias for $\overline{L_{c}}$.

d $\quad \min \left(U-\mu_{c}, \mu_{c}-L\right)$.

$\bar{d} \quad \min \left(U-\bar{\mu}_{c}, \bar{\mu}_{c}-L\right)$.

\section{B. System Analysis}

1) Lead Time Analysis of Delivery Process: In this section, we study the dynamics of material flow in the four-stage supply chain described earlier. This is a classic example of a discrete event dynamical system (a class of systems where the dynamics is completely determined by the occurrence of events at discrete epochs). The various events of the supply chain, that are of our interest, are illustrated in Fig. 7.

In this section, we wish to present a couple of observations about the lead time of the delivery process. These observations will give a sense of end-to-end lead time and will also serve as building block while formulating the IOPT problem. Some of these observations are described in the form of Lemma 1. A few of them, that follow immediately from system dynamics, are stated below without any explanation.

1) End-to-end lead time experienced by manufacturer after placing an order to supplier (i.e., $L_{m}$ ) is given by

$$
\begin{aligned}
L_{m} & =\sum_{i=1}^{2} X_{i} \quad \text { with } \mu_{m}=\sum_{i=1}^{2} \mu_{i} \quad \text { and } \\
\sigma_{m}^{2} & =\sum_{i=1}^{2} \sigma_{i}^{2} .
\end{aligned}
$$

2) The time taken to get finished product ready, after manufacturer places the corresponding order for semifinished product to supplier (i.e., $L_{f}$ ) is given by

$$
L_{f}=\sum_{i=1}^{3} X_{i} \quad \text { with } \mu_{f}=\sum_{i=1}^{3} \mu_{i} \quad \text { and } \quad \sigma_{f}^{2}=\sum_{i=1}^{3} \sigma_{i}^{2} .
$$

The lemma below provides an upper bound on end-to-end lead time experienced by an end user.

Lemma 1: The upper bound on end-to-end lead time $\left(L_{c}\right)$ experienced by an end customer, in a four-stage (LPG) supply chain described above, is given by

$$
\overline{L_{c}}=X_{4}+M_{o}\left(X_{1}+X_{2}+X_{3}\right)
$$

where $M_{o}$ is the stockout probability at the manufacturer (RD).

Proof: Recall that the arrival of an end customer order triggers placement of an order request by the manufacturer to the supplier. Also, the item is shipped to the end customer immediately if it is available in stock at the manufacturer, otherwise the order gets backordered at the manufacturer (see Fig. 7). In the first case, the lead time experienced by the end customer will be the same as the outbound logistics time i.e., $X_{4}$. For the second case, assume that the customer order is the $i$ th backorder at the manufacturer where $i=1,2, \ldots, \infty$. By virtue of the inventory replenishment policy followed by the manufacturer, there will be $(R+i)$ outstanding orders of semifinished products immediately after arrival of this backorder. Remember that it is an underlying assumption of the model that $X_{1}, X_{2}$, and $X_{3}$ are independent across items also. Hence, $L_{f}$ is independent across all these $(R+i)$ orders. It is a direct consequence of this result that the orders placed by the manufacturer can cross each other which means that a product for which supply-chain activities were started later may be ready in finished form earlier than the product for which activities were started earlier. A comprehensive idea of this phenomenon is presented in [22]. 


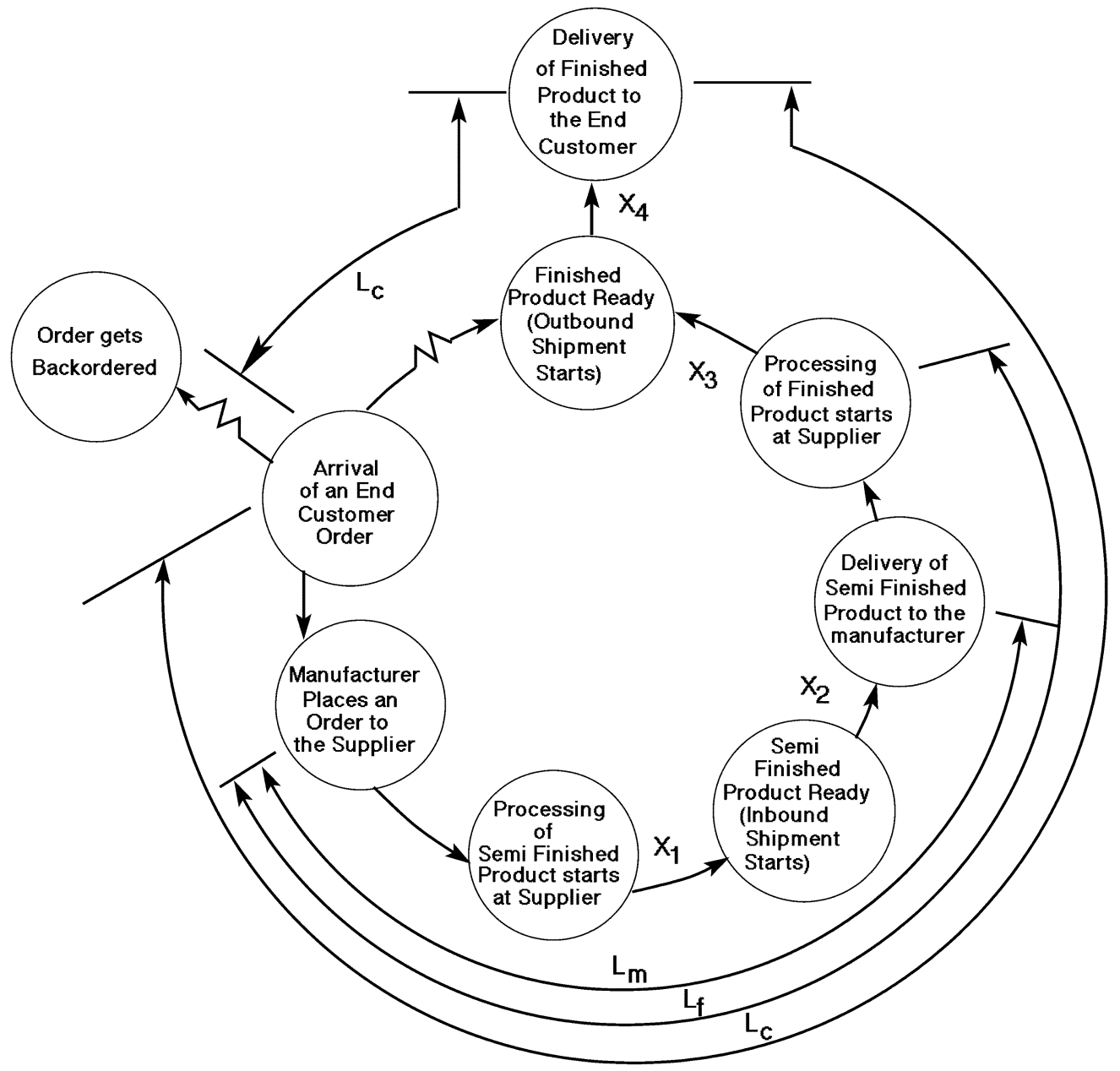

Fig. 7. Event diagram for four-stage supply-chain model.

In view of the crossing of finished products at the manufacturer, it is easy to see that the finished product which is allocated to some backorder may not be the one that results from the corresponding order placed by the manufacturer to the supplier on arrival of this backorder. If it were so, the time taken to serve a backorder by the manufacturer would have been no more than $X_{1}+X_{2}+X_{3}$. Thus, due to:

1) crossing of orders at manufacturer;

2) assumption of indistinguishable products;

3) FIFO policy for serving the backorders;

this time is definitely less than $X_{1}+X_{2}+X_{3}$. Hence it can be said that random variable $X_{1}+X_{2}+X_{3}+X_{4}$ gives an upper bound on the time taken to get the finished product by end customer in the second case.

Considering the first and second case together and using theory of total probability, it is easy to establish following expression for upper bound on lead time experienced by end customer

$$
\begin{aligned}
\overline{L_{c}} & =\left(1-M_{o}\right) X_{4}+M_{o}\left(X_{1}+X_{2}+X_{3}+X_{4}\right) \\
& =X_{4}+M_{o}\left(X_{1}+X_{2}+X_{3}\right) .
\end{aligned}
$$

It is now clear that in the absence of crossover, the end-to-end lead time for the second case will be equal to $X_{1}+X_{2}+X_{3}+X_{4}$. Hence, $\overline{L_{c}}$ will represent the end-to-end lead time $L_{c}$ rather than an upper bound on it. It is interesting to observe that as the variability in lead time or demand process reduces, the likelihood of crossover reduces which in turn brings $\overline{L_{c}}$ closer to $L_{c}$. Formally, it can be said that the difference $\triangle=\overline{L_{c}}-L_{c}$ is a monotonically increasing function of crossover probability $p_{c}$.

2) Analysis of Inventory at the Manufacturing Node: Observe that the manufacturer follows a $\langle Q, R\rangle$ policy, with $Q=1$, for replenishing the finished product inventory (such a policy is also called as the base stock policy in inventory management literature). There is a well-known theorem (after Tackács 1956) [22] for $\langle Q, R\rangle$ models with $Q=1$ which is reproduced below.

Theorem 1: Let the $\langle Q, R\rangle$ policy with $Q=1$ be followed for controlling the inventory of a given item at a single location where the demand is Poisson distributed with rate $\lambda$, and let the replenishment lead times be nonnegative independent random variables (i.e., orders can cross) with density $g(t)$ and mean $\mu$. The steady-state probability of having net inventory (on hand inventory minus backorders) $x$ by such a system can be given by

$$
\psi(x)=\frac{\exp (-\lambda \mu)(\lambda \mu)^{x}}{x !} .
$$

In other words, the steady-state probabilities are independent of the nature of the replenishment lead time distribution if the lead times are nonnegative and independent. 
In the context of the four-stage supply chain, the replenishment lead time for manufacturer is $L_{f}$ which has already been shown to be independent over finished products (i.e., finished products can cross each other). However, $L_{f}$ is a normal random variable which is not nonnegative. Therefore, the above theorem cannot be applied to finished product inventory directly.

Nevertheless, it is safe to assume that the probability of $X_{1}, X_{2}$, and $X_{3}$ taking negative values is small enough that the above theorem can be applied for lead time $L_{f}$ without significant error. For example, if $\mu_{f} \geq 6 \sigma_{f}$ then, the negative area of the probability density function of $L_{f}$ is no more than $10^{-6}$, which can be ignored for all practical purposes and $L_{f}$ can be assumed as virtually nonnegative. In view of this argument, the steady-state probability of having a net inventory $x$ of finished products with the manufacturer can be given as follows:

$$
\begin{aligned}
\psi_{m}(x)=\frac{\exp \left(-\lambda \mu_{f}\right)\left(\lambda \mu_{f}\right)^{x}}{x !} \quad \forall x=R, \\
R-1, \ldots, 0,-1,-2, \ldots
\end{aligned}
$$

Now, it is no more difficult [22] to derive the expressions for the stockout probability $\left(M_{o}\right)$, the average number of backorders per unit time $(E)$, the expected number of backorders at any random instant $(B)$, and the expected number of on-hand inventory at any random instant $(D)$. These expressions are listed as

$$
\begin{aligned}
M_{o} & =P\left(R ; \lambda \mu_{f}\right) \\
& =\sum_{k=R}^{\infty} \frac{\exp \left(-\lambda \mu_{f}\right)\left(\lambda \mu_{f}\right)^{k}}{k !} \\
& =1-\exp \left(-\lambda \mu_{f}\right) \sum_{k=0}^{R-1} \frac{\left(\lambda \mu_{f}\right)^{k}}{k !} \\
E & =\lambda M_{o} \\
B & =\lambda \mu_{f} P\left(R-1 ; \lambda \mu_{f}\right)-R M_{o} \\
& =M_{o}\left(\lambda \mu_{f}-R\right)+\frac{\exp \left(-\lambda \mu_{f}\right)\left(\lambda \mu_{f}\right)^{R}}{(R-1) !} \\
D & =R-\lambda \mu_{f}+B .
\end{aligned}
$$

The expression for $M_{O}$ serves in deriving an important conclusion about upper bound on end-to-end lead time for customer (i.e., $\overline{L_{c}}$ ) which is described (without proof) in the form of Lemma 2.

Lemma 2: For a fixed value of $R, \lambda$, and $\mu_{f}$, the upper bound on end-to-end lead time experienced by an end customer (i.e., $\left.\overline{L_{c}}\right)$ is a normal random variable with mean $\overline{\mu_{c}}$ and variance $\overline{\sigma_{c}}{ }^{2}$ given by

$$
\begin{gathered}
\overline{\mu_{c}}=\mu_{4}+M_{o}\left(\mu_{1}+\mu_{2}+\mu_{3}\right) \\
{\overline{\sigma_{c}}}^{2}=\sigma_{4}^{2}+M_{o}^{2}\left(\sigma_{1}^{2}+\sigma_{2}^{2}+\sigma_{3}^{2}\right)
\end{gathered}
$$

where $M_{O}$ is given by (9).

\section{Formulation of IOPT}

The objective of the study here is to find out how variability should be allocated to the lead times of the individual stages and what should be the optimal value of inventory level $R$, such that the specified levels of DP and DS are achieved for the end customer lead time, in the steady-state condition, in a cost effective manner. We call this problem as the IOPT problem in six sigma supply chains.

It is easy to see that an increase in the value of $R$ results in high inventory carrying cost, and improved quality of deliveries. Similarly, variance reduction of lead time at any stage(s) of the supply-chain results in a high processing cost and improved quality of deliveries. This means that a specified level of quality for the delivery process can be achieved either by increasing the value of $R$ or by reducing the variance of lead time for one or more stages or both. The problem here is to determine a judicious balance between these two such that the cost is minimized.

Depending upon whether $R>0$ or $R=0$, there is a slight change in the formulation of the problem. Therefore, we formulate two separate IOPT problems for the cases $R>0$ (which we call "with stock") and $R=0$ (which we call "with zero stock"). Since in both cases, we use a make-to-order policy to pull the products, we can more completely describe these two policies as make-to-order-with-stock (MTOS) policy and make-to-orderwith-zero-stock (MTOZS) policy, respectively. The input parameters and decision variables are the same for both MTOS Policy and MTOZS Policy. However, the objective function as well as the constraints are different for these two policies. The input parameters, decision variables, objective function, and constraints in the IOPT problem are as follows.

1) Input Parameters: The input parameters to the IOPT problem are: mean $\mu_{i}$ of random variable $X_{i}$, for $i=1,2,3,4$, arrival rate $\lambda$ for customer orders, customer delivery window $(\tau, T)$, desired levels of DP $(\theta \sigma)$ and DS $\left(C_{p m}^{*}\right)$ for customer lead time, and coefficients of the first three terms in Taylor series expansion of processing costs $\mathcal{K}_{i}$ for $i=1,2,3,4$.

It is assumed in Section IV.A2 that $\mathcal{K}_{i}$ is a function of $\mu_{i}$ and $\sigma_{i}$. But $\mu_{i}$ 's are known in the IOPT problem. Therefore, $\mathcal{K}_{i}$ is now a function of $\sigma_{i}$ only. Hence, the first three terms in the Taylor series expansion of $\mathcal{K}_{i}$ can be given as follows:

$$
\mathcal{K}_{i}=A_{i 0}+A_{i 1} \sigma_{i}+A_{i 2} \sigma_{i}^{2}
$$

The coefficients $A_{i 0}, A_{i 1}$, and $A_{i 2}$ do not have an immediate physical significance and will have to be determined by the supply-chain designer using data available about the supplychain business processes.

2) Decision Variables: The decision variables in IOPT are standard deviation $\sigma_{i}$ of each individual stage $i(i=1,2,3,4)$, and the inventory position $R$.

3) Objective Function:

MTOS Policy: We identify the following costs as significant costs for this policy:

- average annual processing cost for supplier $=\lambda \mathcal{K}_{1} \$ /$ year;

- average annual inbound logistics cost $=\lambda \mathcal{K}_{2} \$ /$ year;

- average annual manufacturing cost $=\lambda \mathcal{K}_{3} \$ /$ year;

- average annual outbound logistics cost $=\lambda \mathcal{K}_{4} \$$ /year;

- average annual order placing cost $=\lambda A \$ /$ year;

- average annual backorder cost $=\Pi E+\hat{\Pi} B$ \$/year;

- average annual inventory carrying cost $=I D C_{m}$ \$/year;

- average annual cost of raw material $=\lambda C$ \$/year. 
The sum of all the above mentioned costs gives the total average annual operating cost, say COST. This comes out to be

$$
\mathcal{K}=\lambda \sum_{i=1}^{4} \mathcal{K}_{i}+A \lambda+\Pi E+\hat{\Pi} B+I D C_{m}+\lambda C .
$$

It is easy to see that capital tied up with each unit of finished good, ready to be shipped at manufacturer, includes: raw material cost, total processing cost excluding outbound logistics cost, and order placing cost against this finished good. This comes out to be

$$
C_{m}=C+A+\sum_{i=1}^{3} \mathcal{K}_{i}
$$

This results in

$$
\begin{aligned}
\mathcal{K}=(\lambda+I D) \sum_{i=1}^{3} \mathcal{K}_{i}+\lambda\left(\mathcal{K}_{4}+A+C\right)+\Pi E & \\
& +\hat{\Pi} B+I D(A+C) .
\end{aligned}
$$

Observe that, for a particular value of $R, \lambda, B, D, E, C$, $A, \Pi, \hat{\Pi}, I$ are all known constants and hence they can be combined into one single constant $\beta$ and the equation reduces to

$$
\mathcal{K}=(\lambda+I D)\left(\mathcal{K}_{1}+\mathcal{K}_{2}+\mathcal{K}_{3}\right)+\lambda \mathcal{K}_{4}+\beta
$$

where $\beta=\lambda(A+C)+\Pi E+\hat{\Pi} B+I D(A+C)$. If we use (15) to express $\mathcal{K}_{1}, \mathcal{K}_{2}, \mathcal{K}_{3}, \mathcal{K}_{4}$ then the above expression becomes

$$
\begin{aligned}
\mathcal{K}=(\lambda+I D)\left[\sum_{i=1}^{3}\right. & \left.\left(A_{i 0}+A_{i 1} \sigma_{i}+A_{i 2} \sigma_{i}^{2}\right)\right] \\
& +\lambda\left[A_{40}+A_{41} \sigma_{4}+A_{42} \sigma_{4}^{2}\right]+\beta .
\end{aligned}
$$

MTOZS Policy: This policy is a special case of the previous one in which the inventory carrying cost need not to be considered. The processing costs for each stage in this policy are the same as those of the MTOS Policy because these costs have no relation to the finished product inventory at the manufacturer. Also, raw material cost and order placing cost are the same as in the previous one. However, the expression for the cost of backorders is a little different. Under this policy, every order of the customer which arrives at the manufacturer does not find the finished product and therefore gets backordered there. This implies $M_{o}=1$ so the values of $E$ and $B$ become $\lambda$ and $\lambda \mu_{f}$, respectively. In view of this, the annual backorder cost becomes $\Pi \lambda+\hat{\Pi} \lambda \mu_{f}$. Summing up all the significant costs, we get the following expression for COST in this policy:

$$
\mathcal{K}=\lambda \sum_{i=1}^{4} \mathcal{K}_{i}+A \lambda+\Pi \lambda+\hat{\Pi} \lambda \mu_{f}+\lambda C .
$$

4) Constraints: Recall that $\overline{L_{c}}$ is an upper bound on end customer lead time $L_{c}$. Hence, if we specify the constraints which assure to attain the specified levels of DP and DS for $\overline{L_{c}}$, it will automatically imply that $L_{c}$ attains the same or even better levels of DP and DS than the specified. These constraints can be given as follows:

$$
\begin{aligned}
& \text { DS for } \overline{L_{c}} \geq C_{p m}^{*} \\
& \text { DP for } \overline{L_{c}} \geq \theta \sigma .
\end{aligned}
$$

It is required to express these constraints in terms of decision variables $\sigma_{i}$ 's before we can formulate optimization problem. Recall, Lemma 2 relates the variance ${\overline{\sigma_{c}}}^{2}$ with variances of individual stages, for a given value of $R$ (note that $\lambda$ and $\mu_{f}$ are known here). Thus, for a given value of $R,{\overline{\sigma_{c}}}^{2}$ can be expressed in terms of $C_{p}$ and $C_{p k}$ of $\overline{L_{c}}$ in the following manner:

$$
{\overline{\sigma_{c}}}^{2}=\frac{T^{2}}{9 C_{p}^{2}}=\frac{\bar{d}^{2}}{9 C_{p k}^{2}}
$$

where $T$, the tolerance of customer delivery window, is a known parameter in the IOPT problem and $\bar{d}$ is given as follows:

$$
\bar{d}=\min \left(U-\overline{\mu_{c}}, \overline{\mu_{c}}-L\right) .
$$

Substituting the value of $\overline{\mu_{c}}$, from Lemma 2 in the above relation, we get

$\bar{d}=\min \left(\left[U-\mu_{4}-M_{o} \sum_{i=1}^{3} \mu_{i}\right],\left[\mu_{4}+M_{o} \sum_{i=1}^{3} \mu_{i}-L\right]\right)$.

In the above equation $U, L, \mu_{1}, \mu_{2}, \mu_{3}, \mu_{4}$ are all known parameters. Also, $M_{o}$, according to (9), depends only on $\lambda, R$, and $\mu_{f}$. Therefore, for a given value of $R, \bar{d}$ is a known parameter. The only unknown quantities in (24) are $C_{p}$ and $C_{p k}$. Substituting the value of (24) in (14) we get the following relation which is the crux of the problem of converting constraints in terms of decision variables, for a given value of $R$

$$
\sigma_{4}^{2}+M_{o}^{2}\left(\sigma_{1}^{2}+\sigma_{2}^{2}+\sigma_{3}^{2}\right)=\frac{T^{2}}{9 C_{p}^{2}}=\frac{\bar{d}^{2}}{9 C_{p k}^{2}} .
$$

The above relation is the crux behind expressing the constraints (22), and (23) in terms of decision variables. The idea is like this: choose the values of index $C_{p}$ and $C_{p k}$ for $L_{c}$ in such a way that desired level of DP and DS are ensured. Use these values in above relation. This idea is elaborated in the next section. Under the MTOZS policy, the above expression remains the same except that $M_{o}=1$.

\section{Solution of IOPT}

Observe that the objective function $\mathcal{K}$ is a function of $\sigma_{i}$ 's and $B, D, E$ which are all functions of $R$. Theoretically, $R$ can take any value from set of natural numbers and $\sigma_{i}$ 's can take any positive real value. It makes the optimization problem a mixed integer nonlinear optimization problem. Fortunately, $R$ cannot take any arbitrarily large value. For example, a seasoned asset manager who is engaged in managing the inventory can tell by his experience that $R$ can never exceed a certain value. Also, often times, there is a constraint on storage space, or capital tied up with inventory, etc. which further limits the value of $R$. Therefore, a good way of solving the IOPT problem would be the following.

1) Fix a value for $R$ and solve the resulting subproblem to determine optimal values of $\sigma_{i}$ 's to achieve the optimal COST for that value of $R$. This requires a careful study and interpretation of the constraints to determine the values of $C_{p}$ and $C_{p k}$ for a given value of $R$. This is discussed in the next subsection.

2) Repeat Step 1 for all possible values of $R>0$.

3) Repeat Step 1 for $R=0$. The case $R=0$ is a bit different from that of $R>0$ since it leads to a subtly dif- 
CASE 1
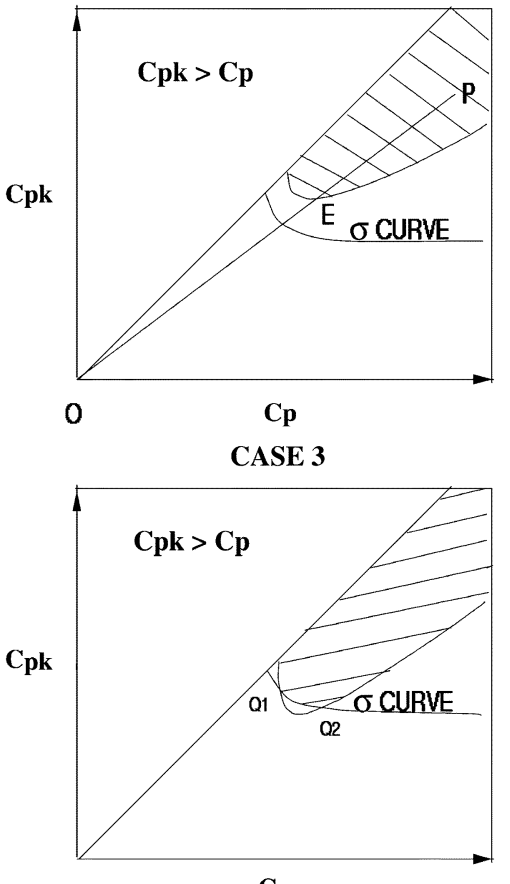

CASE 2
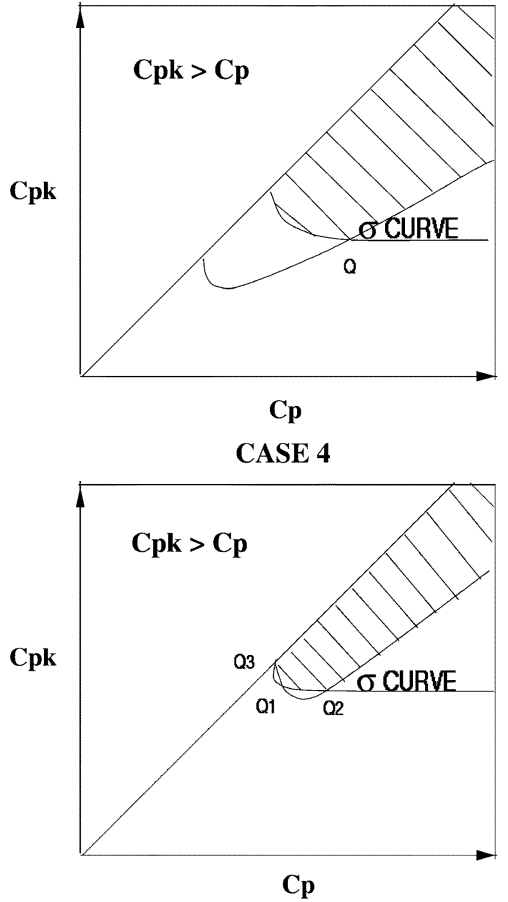

CASE 2

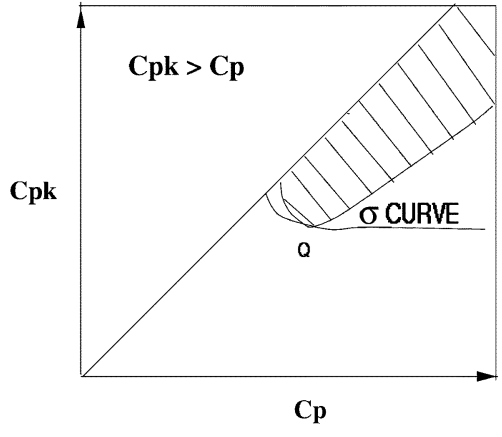

Fig. 8. Possible geometric shapes of feasible region for $C_{P}$ and $C_{p k}$ of $\overline{L_{c}}$.

ferent objective function and subtly different constraints (for details, see [21]).

4) Determine the minimum among all such optimal upper bounds on COST computed above. The corresponding $R$ will give the optimal inventory level to be maintained and the corresponding $\sigma_{i}$ 's will give the optimal variabilities to be assigned to individual lead times.

Thus, the complexity of the IOPT problem will be equal to $O\left(\left(R^{*}+1\right) \times \xi\right)+O\left(R^{*} \log R^{*}\right)$ where $R^{*}$ is the upper bound on inventory $R$ and $\xi$ is the complexity for solving the optimization problem for a fixed value of $R$. Thus, the first term of this expression gives the complexity involved in executing the Steps 1 through 3 of the above algorithm and second term represents the complexity of the Step 4.

\section{E. Determining $C_{p}$ and $C_{p k}$ for a Given Value of $R$}

The unknown pair $\left(C_{p}, C_{p k}\right)$ in (26) is chosen in a way that it satisfies both the constraints (22) and (23). The idea behind getting such a pair is as follows. The relation (26) forces the desired $\left(C_{p}, C_{p k}\right)$ pair to lie on the line $C_{p k}=(\bar{d} / T) C_{p}$ in the $C_{p}-C_{p k}$ plane. Also, it is easy to see that the constraint (22) forces the desired pair to lie on or above the curve $C_{p m}=C_{p m}^{*}$ in $C_{p}-C_{p k}$ plane. Similarly, constraint (23) forces it to lie on or above the $\theta \sigma$ curve in the same plane. All these result in a feasible region in the $C_{p}-C_{p k}$ plane. Fig. 8 shows all possible geometries for such a feasible region, depending upon the relative position of $C_{p m}=C_{p m}^{*}$ curve and the $\theta \sigma$ curve. From Fig. 8, it is clear that the feasible region in each case is the part of the line $C_{p k}=(\bar{d} / T) C_{p}$, denoted by EP, which intersects the shaded region. For the sake of clarity, we have shown the line OP only in Case 1. In all other cases it is understood. Each point of the feasible region satisfies both constraints (22) and
(23) and therefore, can be used as a design point in (26). The concern here is which point should be selected as design point. Before we investigate further in this direction, let us consider a few interesting facts about such a $\left(C_{p}, C_{p k}\right)$ pair in the form of two Lemmas $(3,4)$. These two lemmas are proved in [19] and we reproduce the proofs here for the sake of self-sufficiency.

Lemma 3: For given values of $T$ and $\bar{d}$, DS of $\overline{L_{c}}$ is bounded above by

$$
\overline{C_{p m}}=\frac{T}{3(T-\bar{d})} .
$$

Proof: For a given value of $T$ and $\bar{d}, C_{p}$, and $C_{p k}$ of the process $\overline{L_{c}}$ need to satisfy the following relation (see (26)):

$$
C_{p k}=\left(\frac{\bar{d}}{T}\right) C_{p}
$$

If we take any point on this line, it represents a unique combination of $C_{p}, C_{p k}$, and $C_{p m}$. Hence, if we choose this point as design point, the DS for $\overline{L_{c}}$ gets fixed. Now, consider the following equation for a typical $C_{p m}$ curve on the $C_{p}-C_{p k}$ plane:

$$
\frac{1}{C_{p m}^{2}}=\frac{1}{C_{p}^{2}}+9\left(1-\frac{C_{p k}}{C_{p}}\right)^{2} .
$$

It can be verified that this equation represents a hyperbola. It is quite possible that the line given by (27) becomes an asymptote of such a hyperbola. Such a hyperbola is the curve of $\overline{C_{p m}}$ because it is clear from the geometry of the figure that this line cannot intersect any other $C_{p m}$ curve which is greater than $\overline{C_{p m}}$. Hence, it is not possible to achieve the $C_{p m}$ value (or DS) higher than $\overline{C_{p m}}$ for process $\overline{L_{c}}$, under the given $T$ and $\bar{d}$.

It is easy to show that the slope of asymptotes of $\overline{C_{p m}}$ curve is $\left(1 \pm(1) /\left(3 \overline{C_{p m}}\right)\right)$. Equating these to the slope of the line (27) we get the required expression for $\overline{C_{p m}}$. 


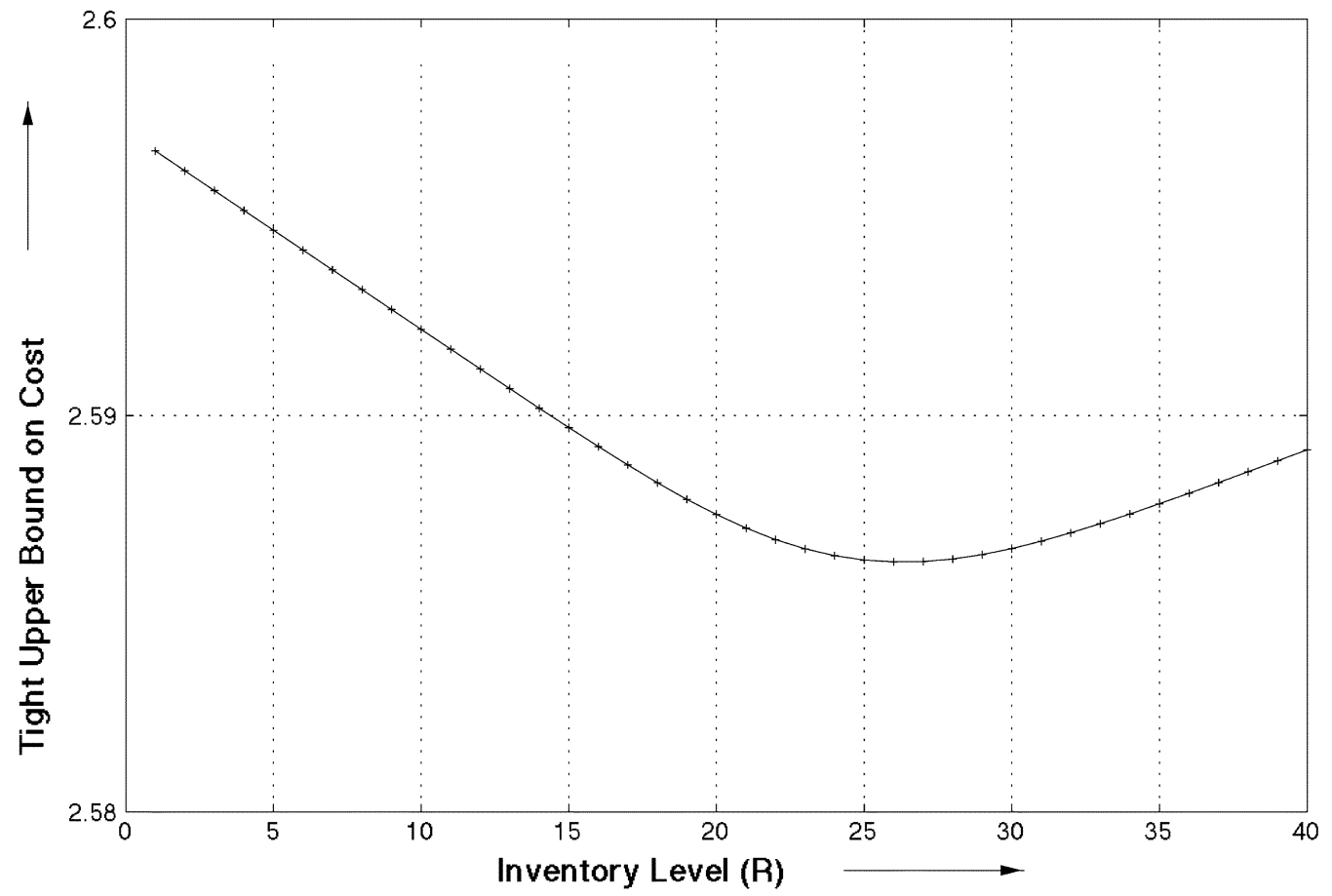

Fig. 9. Optimal inventory level $R$ for $\mathrm{DP}=3 \sigma$ and $\mathrm{DS}=0.7$.

Lemma 4: For given values of $T$ and $\bar{d}$, a unique value of DP gets fixed automatically for $\overline{L_{c}}$ whenever it is attempted to fix DS for $\overline{L_{c}}$ and also vice versa. Moreover, these DP and DS have a positive correlation.

Proof: Earlier, we said that the $\left(C_{p}, C_{p k}\right)$ pair is chosen for $\overline{L_{c}}$ in a way that apart from satisfying both the constraints (22) and (23), the pair must lie on the line (27).

It is easy to verify that a unique $C_{p m}$ curve and a unique $\theta \sigma$ curve pass through a unique point of the line (27). These $\theta \sigma$ values and $C_{p m}$ values are final DP and DS, respectively, which are achieved for $\overline{L_{c}}$ if this particular point is chosen as design point. Hence, it can be concluded that once a value is chosen for DP of $\overline{L_{c}}$, it will automatically decide the corresponding value of DS and also vice versa. To prove the other statement of the lemma, observe that as we move from point $C_{p}=0$ to point $C_{p}=\infty$ on the line (27), the values of both $C_{p m}$ curve and $\sigma$ curve which pass through that point increase. Therefore, DP increases (or decreases) as DS increases (or decreases) for given values of $T$ and $\bar{d}$.

The implication of Lemma 1 is as follows. If the desired $C_{p m}^{*}$ is greater than $\overline{C_{p m}}$ for given values of $T$ and $\bar{d}$, then, the problem is infeasible. In such a situation, we need not proceed any further. Lemma 2 also has a key implication on the problem of fixing the values of $C_{p}$ and $C_{p k}$ for $\overline{L_{c}}$. According to Lemma 2 , DP and DS of $\overline{L_{c}}$ get fixed immediately as soon as a feasible point from line (27) is chosen as design point. It is easy to see that each point on the $C_{p k}-C_{p}$ plane is unique on its own because it has a unique combination of DP and DS. Therefore, it is quite possible that the point which we have chosen results in either higher DP or higher DS than required for the end-to-end delivery process. Hence, it can be claimed that it is not always true that the DP and DS obtained for $\overline{L_{c}}$ from design are exactly the same as given in constraints (22) and (23).
In view of the above findings, the problem of fixing the values of $C_{p}$ and $C_{p k}$ can be addressed as follows. First step toward this is to test the feasibility of the problem through Lemma 1. If the problem turns out to be feasible then each point in the feasible region is allowed to be chosen as design point. However, depending upon the point which is chosen as design point, the final cost $\mathcal{K}^{*}$ (which we get out of solving the optimization problem) may vary. At this point, we cannot say which feasible point will result in minimum cost. Hence, the problem is handled in an indirect manner. The proposed scheme is like this. First, solve the optimization problem without any constraint and get the optimal variance $\bar{\sigma}^{g}$ for $\overline{L_{c}}$. It will result in a global minimum cost. Now, use this variance $\bar{\sigma}^{g}$ to get $C_{p}^{g}$ and $C_{p k}^{g}$ for $\overline{L_{c}}$ which result in minimum cost. If the point $\left(C_{p}^{g}, C_{p k}^{g}\right)$ falls in the feasible region, then, this point is used as a design point $\left(C_{p}^{*}, C_{p k}^{*}\right)$; otherwise, the point $E$ where the line OP enters the shaded region is taken as the final desired $\left(C_{p}^{*}, C_{p k}^{*}\right)$ pair. The reason behind choosing point $E$ as design point is as follows. The values DP and DS which result from point $E$ are the minimum possible values satisfying both the constraints (22) and (23). If we choose any other feasible point, then, even though the resulting DP and DS for $\overline{L_{c}}$ will satisfy the constraints (22) and (23), yet their values will be a bit high and this will lead to higher cost. In this way, we convert the constraints in terms of decision variables for a given value of $R$.

An important point to note here is that (14) holds true only when the negative area of $L_{f}$ is negligibly small. In order for this condition to hold, it is necessary that the following constraint must also be satisfied along with constraint (26):

$$
\begin{aligned}
\mu_{f} & \geq 6 \sigma_{f} \\
\Rightarrow\left(\sigma_{1}^{2}+\sigma_{2}^{2}+\sigma_{3}^{2}\right) & \leq \frac{\left(\mu_{1}+\mu_{2}+\mu_{3}\right)^{2}}{36} .
\end{aligned}
$$


The following equality and inequality constraints are now ready for the IOPT problem:

$$
\begin{aligned}
\sigma_{4}^{2}+M_{o}^{2}\left(\sigma_{1}^{2}+\sigma_{2}^{2}+\sigma_{3}^{2}\right) & =\frac{T^{2}}{9 C_{p}^{2}}=\frac{\bar{d}^{2}}{9 C_{p k}^{2}} \\
\left(\sigma_{1}^{2}+\sigma_{2}^{2}+\sigma_{3}^{2}\right) & \leq \frac{\left(\mu_{1}+\mu_{2}+\mu_{3}\right)^{2}}{36} \\
\sigma_{i} & >0 \quad \forall i=1,2,3,4 .
\end{aligned}
$$

The MTOZS policy is a special case of the MTOS Policy with $R=0$. Under this policy, $M_{o}=1$. Therefore, the constraint (30) remains the same except $M_{o}=1$. However, the constraint (31) is no more needed because in this case $M_{o}=1$ irrespective of whether $\overline{L_{c}}$ is nonnegative or not. Following are the two insights about these constraints.

- Because the formulated constraints ensure the provided levels of DP and DS for $\overline{L_{c}}$ rather than for $L_{c}$, the minimum cost which we get after solving this problem is actually greater than or equal to what is actually required to achieve the specified levels of DP and DS for $L_{c}$. In other words, it can be said that the COST which we get here is an optimal upper bound on the COST for achieving specified levels of DP and DS on $L_{c}$.

- If one looks at the purpose of constraint (31), then it is easy to see that it enforces nonnegativity of $L_{f}$ without which it is not possible to use formula (9) for $M_{o}$. We imposed condition $\mu_{f} \geq 6 \sigma_{f}$ to ensure this nonnegativity and because of that only we got this constraint. We could have as well chosen $\mu_{f} \geq 5 \sigma_{f}$ but in that case error involved in computing the $M_{O}$ with the help of formula (9) would have been higher. The maximum error that we can tolerate in the results will determine whether or not this constraint is needed in the ultimate analysis. If the constraint is removed, the optimization problem will turn out to have only equality constraints.

Having obtained a pair $\left(C_{p}, C_{p k}\right)$ for the given $R$, we will thus be required to solve a nonlinear optimization problem with equality constraints. The Lagrange multiplier method can be used here.

\section{F. Solution of IOPT for a Specific Instance}

Let us consider the LPG supply chain once again and study the problem in a realistic setting. We have chosen the following values for typical known parameters of the IOPT problem in the context of the LPG supply chain. The values we have chosen.

1) Lead Time Parameters: $\mu_{1}=1$ day, $\mu_{2}=3$ days, $\mu_{3}=2$ days, and $\mu_{4}=7$ days.

2) Demand Process Parameters: $\lambda=1500$ trucks/year.

3) Cost Parameters: These parameters have been chosen so as to capture the negative correlation between cost and mean lead time and between cost and variability of lead time

$$
\begin{aligned}
& \mathcal{K}_{1}=10\left(1+\exp \left(\frac{1}{\sigma_{1}}\right)-\frac{\mu_{1}}{200}\right) \$ / \text { truck } \\
& \mathcal{K}_{2}=100\left(1+\exp \left(\frac{1}{\sigma_{2}}\right)-\frac{\mu_{2}}{200}\right) \$ / \text { truck } \\
& \mathcal{K}_{3}=10\left(1+\exp \left(\frac{1}{\sigma_{3}}\right)-\frac{\mu_{3}}{200}\right) \$ / \text { truck }
\end{aligned}
$$

$$
\begin{aligned}
\mathcal{K}_{4} & =100\left(1+\exp \left(\frac{1}{\sigma_{4}}\right)-\frac{\mu_{4}}{200}\right) \$ / \text { truck } \\
A & =5 \$ / \text { order } \quad \Pi=0 \$ / \text { truck; } \\
\hat{\Pi} & =500 \$ / \text { truck}- \text { year } \\
I & =0.2 \$ / \text { year }-\$ \text { invested } \\
C & =1000 \$ / \text { truck. }
\end{aligned}
$$

4) Delivery Quality Parameters: $\tau=10$ days; $T=10$ days For the sake of numerical experimentations, we consider the following four different sets of constraints and solve the problem under each case:

1) $\mathrm{DP}=3 \sigma$ and $\mathrm{DS}=0.7$ for $L_{c}$ (see Fig. 9);

2) $\mathrm{DP}=4 \sigma$ and $\mathrm{DS}=0.8$ for $L_{c}$ (see Fig. 10);

3) $\mathrm{DP}=5 \sigma$ and $\mathrm{DS}=0.9$ for $L_{c}$ (see Fig. 11);

4) $\mathrm{DP}=6 \sigma$ and $\mathrm{DS}=1.0$ for $L_{c}$ (see Fig. 12).

Assume that it is not possible for the RD to keep more than 40 LPG trucks ready at any given point of time.

We first describe Step 1 of the procedure to solve IOPT, discussed in the last section, for this numerical example. Let us choose constraint set DP $=3 \sigma$ and DS $=0.7$ to work with. Step 2 can be carried out in the same manner for all the other values of $R$. Step 3 and Step 4 are also trivial. The same procedure can be repeated for other constraints sets also.

To start with, let us fix $R=10$. We first compute the following parameters for the given numerical values:

$$
\begin{aligned}
\mu_{f} & =6 \text { days } \\
M_{o} & =0.999722639663766 \\
E & =1499.583959 \text { trucks/year } \\
B & =14.657947016303742 \text { trucks } \\
D & =0.000412769728402651 \text { trucks } \\
\beta & =4.983672090063828 \times 10^{6} \\
\bar{d} & =7.001664162017404 \text { days }
\end{aligned}
$$

Substitution of these values in (20) and (30) results in the following optimization problem:

$$
\begin{aligned}
& \text { Minimize } \\
& \begin{aligned}
\mathcal{K}= & 1500.000083 \sum_{i=1}^{3}\left(A_{i 0}+A_{i 1} \sigma_{i}+A_{i 2} \sigma_{i}^{2}\right) \\
& +1500\left[A_{40}+A_{41} \sigma_{4}+A_{42} \sigma_{4}^{2}\right]+4.983672 \times 10^{6} \\
& \quad\left(\text { say }, \mathcal{K}=a+\sum_{i=1}^{4} a_{i 1} \sigma_{i}+a_{i 2} \sigma_{i}^{2}\right)
\end{aligned}
\end{aligned}
$$

where

$$
\begin{aligned}
& a_{11}=1500.000083 A_{11} \\
& a_{12}=1500.000083 A_{12} \\
& a_{21}=1500.000083 A_{21} \\
& a_{22}=1500.000083 A_{22} \\
& a_{31}=1500.000083 A_{31} \\
& a_{32}=1500.000083 A_{32} \\
& a_{41}=1500 A_{41} \\
& a_{42}=1500 A_{42}
\end{aligned}
$$




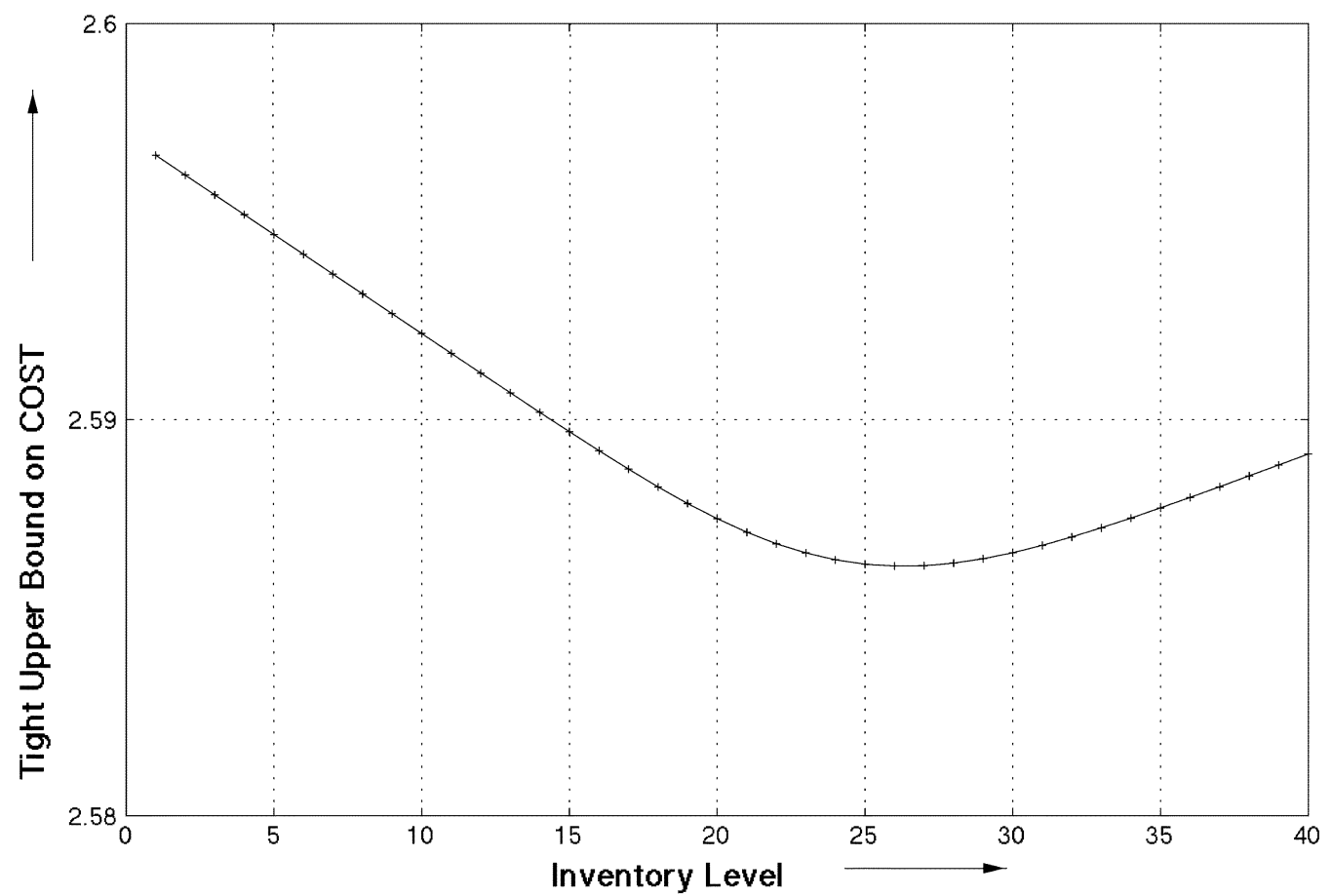

Fig. 10. Optimal inventory level $R$ for $\mathrm{DP}=4 \sigma$ and $\mathrm{DS}=0.8$

$$
a=1500.000083 \sum_{i=1}^{3} A_{i 0}+1500 A_{40}+4.983672 \times 10^{6}
$$

subject to

$$
\begin{aligned}
\sigma_{4}^{2}+0.999445\left(\sigma_{1}^{2}+\sigma_{2}^{2}+\sigma_{3}^{2}\right)= & \frac{100}{9 C_{p}^{2}}=\frac{49.0233}{9 C_{p k}^{2}} \\
& \sigma_{i}>0 \quad \forall i=1,2,3,4 .
\end{aligned}
$$

The constants $A_{i j}$ can be determined with the help of Taylor series expansion of the cost functions $\mathcal{K}_{i}$. We have expanded all the cost functions at $\sigma_{i}=1$ and used the corresponding coefficients as the constants $A_{i j}$. The immediate problem is to find out values of $C_{p}$ and $C_{p k}$. As a first step toward this, it is required to check the feasibility of the problem as per guidelines provided in Lemma 1. Note the upper bound on DS for this case is

$$
\overline{C_{p m}}=\frac{T}{3(T-\bar{d})}=1.111727809 .
$$

Hence, as far as feasibility is concerned, there is no problem because all the desired values of DS are within permissible range. As a next step we find out the pair $C_{p}^{g}$ and $C_{p k}^{g}$ that results in global minimum and test whether it belongs to the feasible region or not. For this, let us assume that $S=\left\{\left(\sigma_{1}, \sigma_{2}, \sigma_{3}, \sigma_{4}\right)\right.$ : $\left.\sigma_{i} \in \Re^{+} \forall i=1,2,3,4\right\}$. It immediately follows from this definition of $S$ that $\mathcal{K}: S \rightarrow E_{1}$ where $S$ is a nonempty open convex set. To test the convexity of objective function $\mathcal{K}$, we compute gradient vector $\nabla \mathcal{K}(\overline{\mathbf{X}})$ and Hessian matrix $H(\overline{\mathbf{X}})$ for function $\mathcal{K}$ at point $\overline{\mathbf{X}}=\left(\sigma_{1}, \sigma_{2}, \sigma_{3}, \sigma_{4}\right)^{T}$. Observe that the gradient vector and Hessian exist for each $\overline{\mathbf{X}} \in S$. It directly follows that function $\mathcal{K}$ is twice differentiable over $S$. Moreover, the Hessian is independent of $\overline{\mathbf{X}}$. Therefore, it is sufficient that we test the positive definiteness (PD) or positive semidefi- niteness (PSD) of the Hessian at any one point of $S$ instead of testing it all over $S$.

It is easy to see that all the diagonal elements of the Hessian are positive real numbers because $A_{i 2}$ are positive. Therefore, the Hessian is PD and function $\mathcal{K}$ is strictly convex which implies that a local optimal solution of unconstrained problem is the unique global optimal solution. This can be obtained by equating $\nabla \mathcal{K}(\overline{\mathbf{X}})$ to 0 . For the present numerical example it results in $\sigma_{1}^{g}=\sigma_{2}^{g}=\sigma_{3}^{g}=\sigma_{4}^{g}=1.333$ days. These $\sigma_{i}^{g}$ can be used to find out $\sigma^{g}$ which comes out to be 2.66544 days. Indexes $C_{p}^{g}$ and $C_{p k}^{g}$ can be computed by using $\sigma^{g}$. For the present example, these indexes are $C_{p}^{g}=1.25057$ and $C_{p k}^{g}=0.875609$. These $C_{p}^{g}$ and $C_{p k}^{g}$ can further be utilized to determine the value of DP and DS at the global minimum point which come out to be $4.12678 \sigma$ and 0.83088 respectively. These quality levels are more than what is desired. Hence, we use $C_{p}^{g}$ and $C_{p k}^{g}$ as design values. If these quality levels come out to be less than specified in the constraints, it is required to use the scheme suggested earlier in Section IV.C.4. Substituting the $\sigma_{i}^{g}$ 's in objective function (33) gives optimal upper bound on COST (2.5921 million \$) of supply chain with $R=10$.

Fortunately, in the present situation the global minimum point becomes a design point so we need not proceed for any further calculation. But if it is not so, then we will be required to solve the underlying optimization problem by the Lagrange multiplier method and get stationary points which satisfy the necessary conditions. This is explained below.

Method of Lagrange Multipliers:

Lagrange Function: The Lagrange function $L\left(\sigma_{1}, \sigma_{2}, \sigma_{3}\right.$, $\left.\sigma_{4}, \nu\right)$ is given as

$L\left(\sigma_{1}, \sigma_{2}, \sigma_{3}, \sigma_{4}, \nu\right)=\mathcal{K}+\nu\left(\sigma_{4}^{2}+0.999445\left(\sum_{i=1}^{3} \sigma_{i}^{2}\right)-\theta\right)$ 


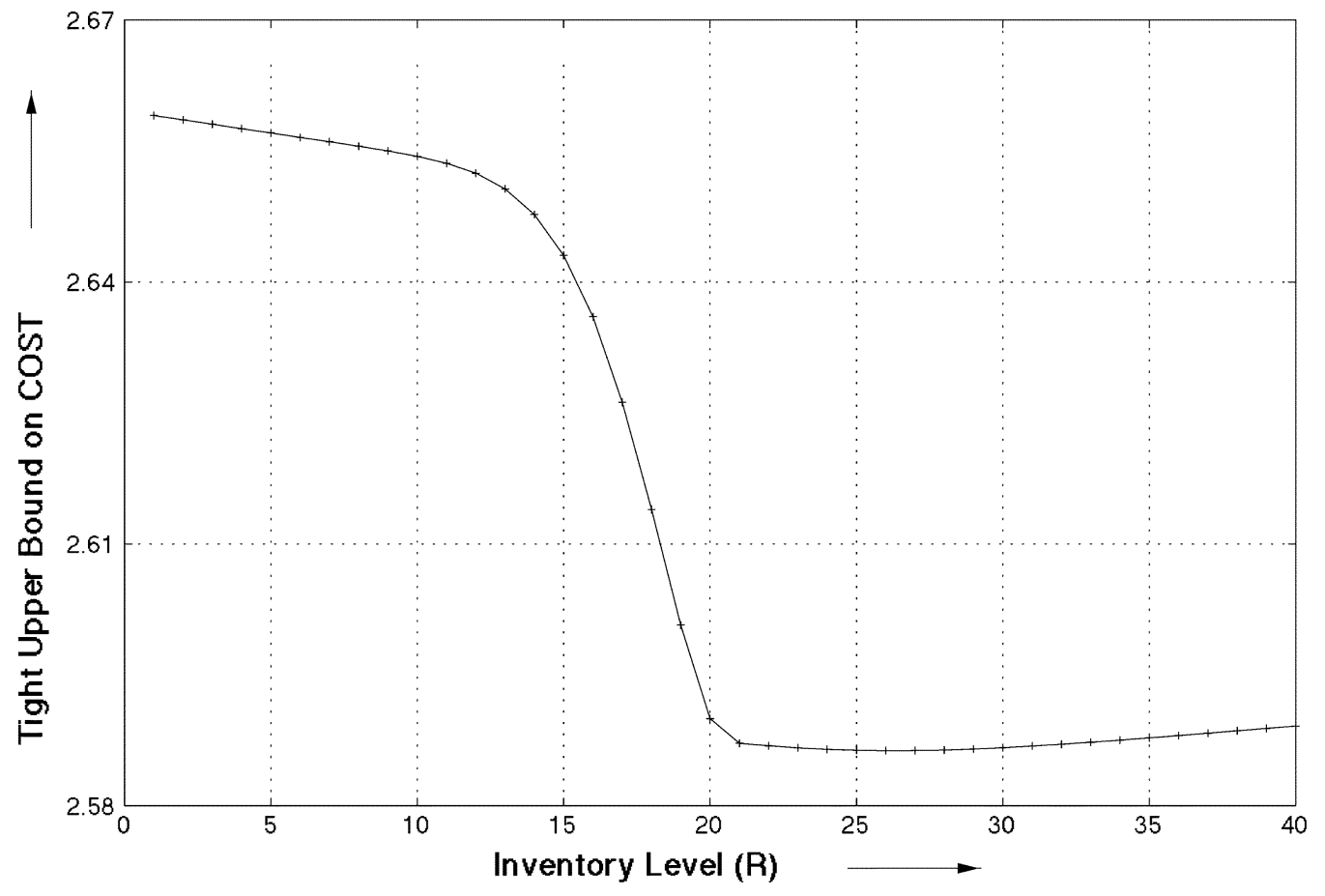

Fig. 11. Optimal inventory level $R$ for $\mathrm{DP}=5 \sigma$ and $\mathrm{DS}=0.9$.

where $\theta=\left(100 / 9 C_{p}^{2}\right)=\left(49.0233 / 9 C_{p k}^{2}\right)$ and $\mathcal{K}$ is given by (33).

Necessary Condition for Stationary Points: Let point $\mathcal{P}^{*}=\left(\sigma_{1}^{*}, \sigma_{2}^{*}, \sigma_{3}^{*}, \sigma_{4}^{*}, \nu^{*}\right)$ correspond to a local optimal point, then this point must satisfy the following necessary conditions for being a stationary point:

$$
\left.\frac{\partial L}{\partial \sigma_{1}}\right|_{\mathcal{P}^{*}}=\left.\frac{\partial L}{\partial \sigma_{2}}\right|_{\mathcal{P}^{*}}=\left.\frac{\partial L}{\partial \sigma_{3}}\right|_{\mathcal{P}^{*}}=\left.\frac{\partial L}{\partial \sigma_{4}}\right|_{\mathcal{P}^{*}}=\left.\frac{\partial L}{\partial \nu}\right|_{\mathcal{P}^{*}}=0 .
$$

These necessary conditions result in the following relations:

$$
\begin{aligned}
\sigma_{1}= & \frac{-a_{11}}{2\left(a_{12}+0.999445 \nu\right)} \\
\sigma_{2}= & \frac{-a_{21}}{2\left(a_{22}+0.999445 \nu\right)} \\
\sigma_{3}= & \frac{-a_{31}}{2\left(a_{32}+0.999445 \nu\right)} \\
\sigma_{4}= & \frac{-a_{41}}{2\left(a_{42}+\nu\right)} \\
\theta= & \frac{0.999445 a_{11}^{2}}{4\left(a_{12}+0.999445 \nu\right)^{2}}+\frac{0.999445 a_{21}^{2}}{4\left(a_{22}+0.999445 \nu\right)^{2}} \\
& +\frac{0.999445 a_{31}^{2}}{4\left(a_{32}+0.999445 \nu\right)^{2}}+\frac{a_{41}^{2}}{4\left(a_{42}+\nu\right)^{2}} .
\end{aligned}
$$

Solving the above system of equations, by some numerical technique, will give the desired stationary points. First of all those stationary points are discarded which are either imaginary or for which the nonnegativity condition does not hold. After this, we apply second order conditions to determine whether the point is a maxima or a minima. Among all the minima points, the one which yields minimum COST is considered as the solution of the problem and we call it as the optimal upper bound on COST for $R=10$ yielding at least $\mathrm{DP}=5 \sigma$, and $\mathrm{DS}=0.7$.

\section{G. Some Numerical Results}

Note that in the last section, we studied an instance of the IOPT problem assuming $R=10, \mathrm{DP}=3 \sigma$, and $\mathrm{DS}=0.7$ for $L_{c}$. We obtained the optimal allocation of standard deviations to achieve a minimum COST. The standard deviations obtained can be used by a supply-chain manager to decide among alternate logistics providers or alternate suppliers, etc.

To obtain the optimal value of $R$, we repeat the solution of the IOPT problem for different values of $R$, each time computing the optimal upper bound on COST and the corresponding allocation of variabilities. The results of the above problem are summarized in the following four plots, one for each set of constraints. Each curve represents the variation of optimal upper bound on COST (\$/year) with inventory level $R$.

One can observe the following trends.

1) The optimal value of inventory is $R^{*}=26$ for the first set of constraints. This optimal value changes for a different set of constraints.

2) The optimal level $R^{*}$ increases as the desired quality level increases. This can be verified by observing the trends of the plots. Notice that for lower values of DP and DS, keeping inventory is always profitable. If desired DP and DS levels are high, the variabilities of the individual processes must be low enough to afford the luxury of having very less inventory. It results in higher cost. In such a situation, higher inventory levels can only allow us to have luxury of high DP and DS.

3) We found in some cases $R^{*}$ turns out to be equal to zero. This means that an inventory less system may be the best option under some circumstances, providing a major case for zero inventories. 


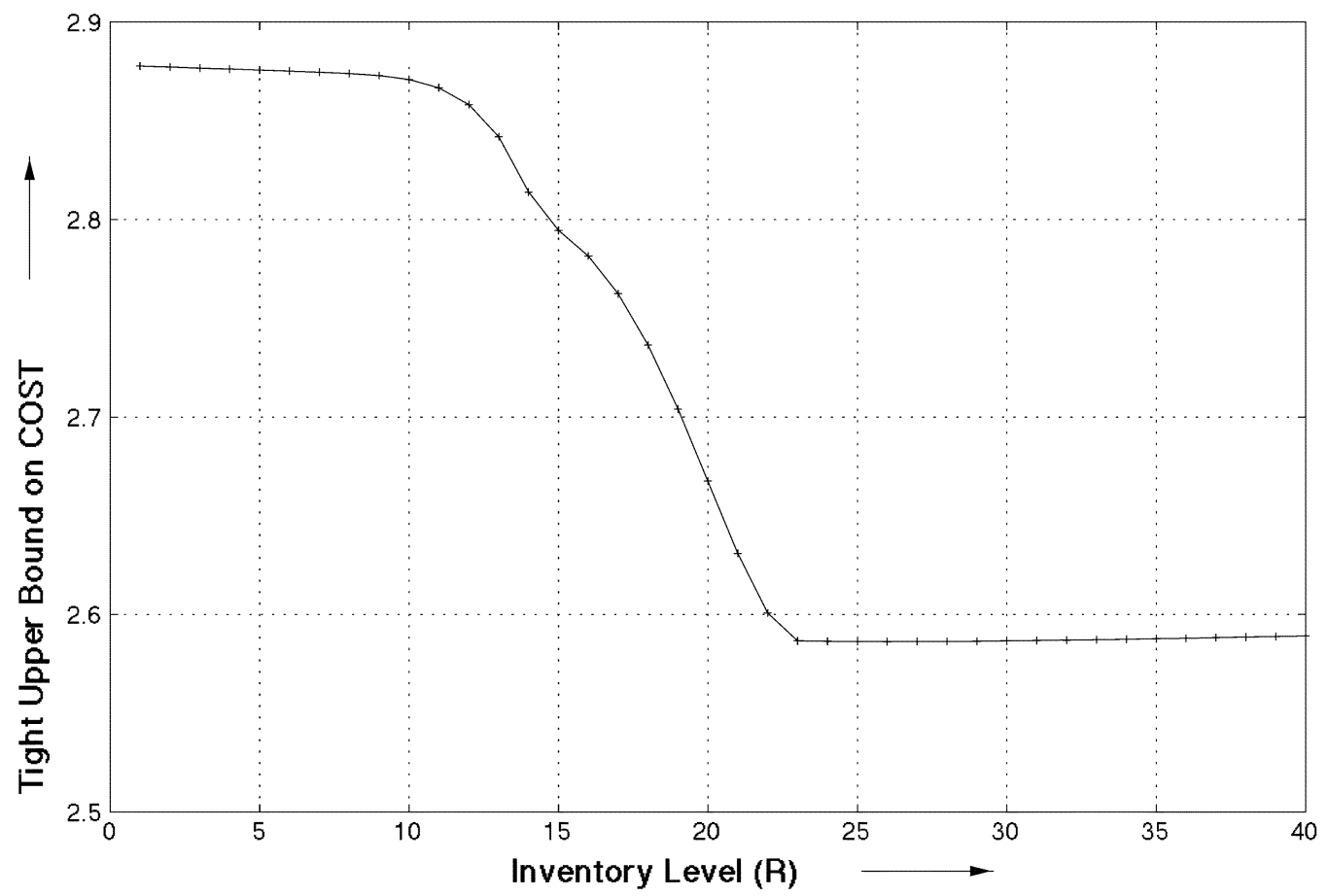

Fig. 12. Optimal inventory level $R$ for $\mathrm{DP}=6 \sigma$ and $\mathrm{DS}=1.0$.

\section{SUMMARY AND FUTURE WORK}

In this paper, we have presented a novel approach to achieve variability reduction, synchronization, and therefore delivery performance improvement in supply-chain networks. Our approach exploits connections between design tolerancing in mechanical assemblies and lead time compression in supply-chain networks. The contributions of this paper can be summarized as follows.

- Defining the notion of six sigma supply chains, to describe supply-chain networks with superior levels of DS and DP.

- Describing design of six sigma supply chains as a mathematical programming problem, thus providing a sound framework for studying a rich variety of design optimization and tactical decision making problems in supply chains

- Illustrating the efficacy of the approach by formulating and solving the IOPT problem for a four-stage LPG supply-chain network.

The paper leaves plenty of room for further work in several directions. The design problem that we studied here is only one of a rich variety of design optimization problems that one can address in the framework developed in this paper. Many other problems, as listed in Section III.F can be studied. Also, the supply-chain example that we have looked at belongs to the make-to-order type and has only one stock. Multiechelon supply chains with multiple inventories and make-to-order policy would be the next category of systems that can be addressed using our methodology. There is no reason why our approach cannot be applied for coordination types other than make-to-order, such as make-to-stock (MTS) and build to order (BTO).

We believe the concepts and approach developed in this paper provide a framework in which a rich variety of supply-chain design and tactical decision problems can be addressed. Developing a sound methodology for rigorous design of complex supply-chain networks is the ultimate goal of this research.

\section{ACKNOWLEDGMENT}

The first two authors would like to deeply appreciate the collaboration with the Manufacturing Systems Research Laboratory, GM R \& D, Warren, MI. In particular, we thank Dr. J. D. Tew, Dr. D. Kulkarni, and Dr. E. Foster of the above laboratory for useful discussions.

\section{REFERENCES}

[1] Y. Narahari, R. Sudarsan, K. Lyons, M. Duffey, and R. Sriram, "Design for tolerancing of electromechanical assemblies: An integrated approach," IEEE Trans. Robot. Automat., vol. 15, pp. 1062-1079, Dec. 1999.

[2] V. E. Kane, "Process capability indices," J. Qual. Technol., vol. 18, pp. 41-52, 1986

[3] S. Kotz and C. R. Lovelace, Process Capability Indices in Theory and Practice. New York: Arnold, 1998.

[4] M. J. Harry, "The nature of six sigma quality," Motorola Inc., Motorola Univ. Press, Schaumburg, IL, Tech. Rep., 1987.

[5] A. Song, A. Mathur, and K. Pattipati, "Design of process parameters using robust design techniques and multiple criteria optimization," IEEE Trans. Syst., Man Cybern., vol. 25, pp. 1437-1446, Nov. 1995.

[6] Y. Narahari, N. Viswanadham, and V. K. Kumar, "Lead time modeling and acceleration of product design and development," IEEE Trans. Robot. Automat., vol. 15, pp. 882-896, Oct. 1999.

[7] W. J. Hopp and M. L. Spearman, Factory Physics: Foundations of Manufacturing Management. New York: McGraw-Hill, 1996.

[8] Y. Narahari, N. Viswanadham, and R. Bhattacharya, "Design of synchronized supply chains: A six sigma tolerancing approach," in Proc. IEEE Int. Conf. Robot. Automat., ICRA'O0, vol. 2, San Francisco, CA, Apr. 2000, pp. 1151-1156.

[9] R. A. Boyles, "The Taguchi capability index," J. Qual. Technol., vol. 23, no. 1, pp. 17-26, 1991.

[10] D. H. Evans, "Statistical tolerancing: The state of the art-Part II: Methods for estimating moments," J. Qual. Technol., vol. 7, no. 1, pp. $1-12,1975$. 
[11] — "Statistical tolerancing: The state of the art-Part III: Shifts and drifts," J. Qual. Technol., vol. 7, no. 2, pp. 72-76, 1975.

[12] M. J. Harry and R. Stewart, "Six sigma mechanical design tolerancing," Motorola Inc., Motorola Univ. Press, Schaumburg, IL, Tech. Rep., 1987.

[13] U. Roy, R. Sudarsan, Y. Narahari, R. D. Sriram, K. W. Lyons, and M. R. Duffey, "Information models for design tolerancing: from conceptual to the detail design," Nat. Inst. Standards Technol., Gaithersburg, MD, Tech. Rep. NISTIR6524, May 2000.

[14] J. M. Masters, "Determination of near optimal stock levels for multiechelon distribution inventories," J. Bus. Logist., vol. 14, no. 2, pp. 165-194, 1993.

[15] M. Ettl, G. E. Feigin, G. Y. Lin, and D. D. Yao, "A supply network model with base-stock control and service requirements," Oper. Res., vol. 48 , no. 2, pp. 216-32, 2000.

[16] S. Tayur, R. Ganeshan, and M. Magazine, Eds., Quantitative Models for Supply Chain Management. Norwell, MA: Kluwer, 1999.

[17] L. B. Schwarz and Z. K. Weng, "The design of a JIT supply chain: The effect of leadtime uncertainty on safety stock," J. Bus. Logist., vol. 21, no. 2 , pp. 231-253, 2000

[18] D. Garg, Y. Narahari, and N. Viswanadham, "Achieving sharp deliveries in supply chains through variance pool allocation," in Proc. IEEE Int. Conf. Robotics Automation (ICRA'02), Washington, DC, May 2002.

[19] - (2002, Aug.) Achieving sharp deliveries in supply chains through variance pool allocation. Electronic Enterprises Lab., Dep. Comp. Sci. Automat., Indian Inst. of Sci., Bangalore, India. [Online]. Available: http://lcm.csa.iisc.ernet.in/Students/garg/garg.html

[20] D. Garg and Y. Narahari, "A process capability indices based approach for supply-chain performance analysis," in Proc. Int. Conf. Energy, Automation Information Technology (EAIT'01). Kharaghpur, India, Dec. 2001.

[21] D. Garg. (2002, May) Design of six sigma supply chains. Master's dissertation, Dept. Comp. Sci. Automat., Indian Inst. Sci., Bangalore, India. [Online]. Available: http://lcm.csa.iisc.ernet.in/Students/garg/garg.html

[22] G. Hadley and T. M. Whitin, Analysis of Inventory Systems. Englewood Cliffs, NJ: Prentice Hall, 1963.

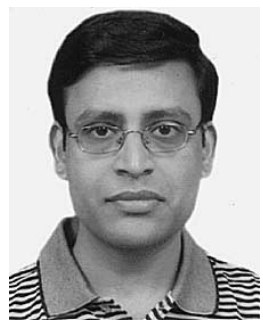

D. Garg (S'02) received the M.Sc. (Eng.) degree in computer science and automation, in 2002, from the Indian Institute of Science, Bangalore, India, where he is currently working toward the Ph.D. degree.

His current research interests include supply-chain management, electronic commerce, mechanism design, and game theory.

Mr. Garg was awarded the Infosys Trophy for Excellence in Management Research in COSMAR-2002 and the Best Presentation Award for Perspective Seminar Series held at Department of Computer Science and Automation, Indian Institute of Science, Bangalore, during January-April 2003.

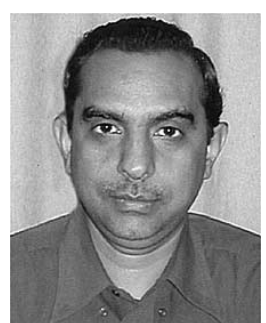

Y. Narahari (SM'02) received the M.E. and Ph.D. degrees in computer science from the Indian Institute of Science, Bangalore, India, in 1984 and 1988, respectively.

He is currently a Professor in the Department of Computer Science and Automation, Indian Institute of Science. His current research interests are in electronic commerce, auction and mechanism design, dynamic pricing models, supply-chain design and optimization, and software architecture for e-business. In 1992, he was a Visiting Researcher on sabbatical at the Massachusetts Institute of Technology, Cambridge, and in 1997, at the National Institute of Standards and Technology, Gaithersburg, MD. He has consulted for several companies including Intel, General Motors Research, WIPRO, HCL, Satyam, and Tektronix. He has coauthored a widely acclaimed textbook Performance Modeling of Automated Manufacturing Systems, (Englewood Cliffs, NJ: Prentice-Hall, 1992) and has recently completed a very popular web-based text on data structures and algorithms.

Prof. Narahari has been on the editorial board of IEEE TRANSACTIONS ON ROBOTICS AND AUTOMATION, and is currently on the editorial board of IEEE TRANSACTIONS ON SYSTEMS, MAN, AND CYBERNETICS. The awards secured by Prof. Narahari include: the Indian Institute of Science Best Ph.D. Thesis Award in 1988; the Indo-US Science and Technology Fellowship in 1992; and the Sir C. V. Raman Young Scientist Award for Computer Science Research, in 1998

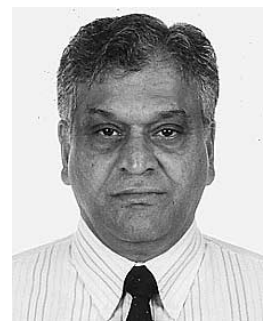

N. Viswanadham (F'93) is Deputy Executive Director of The Logistics Institute-Asia Pacific, Singapore, and also a Professor in Department of Mechanical and Production Engineering, National University of Singapore (NUS), Singapore. He was a Tata Chemicals Chair Professor at the Indian Institute of Science, Bangalore, India. He has contributed significantly to the area of automation, and, in particular, manufacturing automation. $\mathrm{He}$ is the author of three textbooks, six edited volumes, over 75 journal articles, and more than a 100 conference papers on automation. He is the lead author of the textbook entitled Performance Modeling of Automated Manufacturing Systems, (Englewood Cliffs, NJ: Prentice-Hall, 1992). His recent book is entitled Analysis of Manufacturing Enterprises-An approach to leveraging the value delivery processes for competitive advantage (Norwell, MA: Kluwer, 2000). Apart from his widely followed books, he has published a number of high-impact papers on quantitative performance analysis of production, product development, logistics and supply-chain processes of a manufacturing enterprise. His current research interests include logistics, supply-chain networks, and manufacturing systems, and he is developing a methodology for design of six-sigma, synchronized manufacturing and service supply-chain networks. His research has very strong practical as well as theoretical flavors and impact.

Prof. Viswanadham was a GE Research Fellow during 1989-1990 and the recipient of the 1996 Indian Institute of Science Alumni award for excellence in research. He is an Editor of IEEE TRANSACTIONS ON ROBOTICS AND AUTOMATION and the IEEE TRANSACTIONS ON AUTOMATION SCIENCES AND ENGINEERING. $\mathrm{He}$ is also a Fellow of the Indian National Science Academy, Indian Academy of Sciences, Indian National Academy of Engineering, and the Third World Academy of Sciences 